\title{
COMBINED MODELING OF ACCELERATION, TRANSPORT, AND HYDRODYNAMIC RESPONSE IN SOLAR FLARES. II. INCLUSION OF RADIATIVE TRANSFER WITH RADYN
}

\author{
Fatima Rubio da Costa ${ }^{1}$, Wei Liu ${ }^{2,3,4}$, Vahé Petrosian ${ }^{1}$, and Mats Carlsson ${ }^{5}$ \\ ${ }^{1}$ Department of Physics, Stanford University, Stanford, CA 94305, USA; frubio@ stanford.edu \\ ${ }^{2}$ W. W. Hansen Experimental Physics Laboratory, Stanford University, Stanford, CA 94305, USA \\ ${ }^{3}$ Lockheed Martin Solar and Astrophysics Laboratory, 3251 Hanover Street, Building 252, Palo Alto, CA 94304, USA \\ ${ }^{4}$ Bay Area Environmental Research Institute, 625 2nd Street, Suite 209, Petaluma, CA 94952-5159 \\ ${ }^{5}$ Institute of Theoretical Astrophysics, University of Oslo, P.O. Box 1029 Blindern, NO-0315 Oslo, Norway \\ Received 2015 May 5; accepted 2015 August 25; published 2015 November 9
}

\begin{abstract}
Solar flares involve complex processes that are coupled and span a wide range of temporal, spatial, and energy scales. Modeling such processes self-consistently has been a challenge in the past. Here we present results from simulations that couple particle kinetics with hydrodynamics (HD) of the atmospheric plasma. We combine the Stanford unified Fokker-Planck code that models particle acceleration and transport with the RADYN HD code that models the atmospheric response to collisional heating by accelerated electrons through detailed radiative transfer calculations. We perform simulations using two different electron spectra, one an ad hoc power law and the other predicted by the model of stochastic acceleration by turbulence or plasma waves. Surprisingly, the later model, even with energy flux $\ll 10^{10} \mathrm{erg} \mathrm{s}^{-1} \mathrm{~cm}^{-2}$, can cause "explosive" chromospheric evaporation and drive stronger up- and downflows (and HD shocks). This is partly because our acceleration model, like many others, produces a spectrum consisting of a quasi-thermal component plus a power-law tail. We synthesize emission-line profiles covering different heights in the lower atmosphere, including $\mathrm{H} \alpha 6563 \AA$, He II $304 \AA$, Ca II K $3934 \AA$, and Si Iv $1393 \AA$. One interesting result is the unusual high temperature (up to a few times $10^{5} \mathrm{~K}$ ) of the formation site of He II $304 \AA$, which is expected owing to photoionization-recombination under flare conditions, compared to those in the quiet Sun dominated by collisional excitation. When compared with observations, our results can constrain the properties of nonthermal electrons and thus the poorly understood particle acceleration mechanism.
\end{abstract}

Key words: acceleration of particles - hydrodynamics - line: profiles - radiative transfer - Sun: chromosphere Sun: flares

Supporting material: animation

\section{INTRODUCTION}

One of the outstanding problems in solar physics is how magnetic energy is transformed into the observed signatures of solar flares. It has been recently recognized that modeling the coupling between the particle acceleration and transport processes in solar flares and the dynamical response of the atmosphere to particle collisional heating is critical to our understanding of flare dynamics. It is clear that nonthermal electrons and ions play an important role. However, the exact mechanism of acceleration of these particles is still a matter of considerable debate. Several scenarios have been proposed, and different models have been developed with different degrees of details. Among these are acceleration by DC electric fields (e.g., Holman 1985), shocks (e.g., Tsuneta \& Naito 1998), and turbulence. Stochastic acceleration (SA) by turbulence or plasma waves (Ramaty 1979; Hamilton \& Petrosian 1992; Miller 1997) has been developed in greater detail (Petrosian \& Liu 2004) and has been tested by observations more rigorously (Petrosian \& Donaghy 1999; Liu et al. 2004, 2006); for a recent review see Petrosian (2012).

Observations most intimately connected to the acceleration process are the microwave, hard X-ray (HXR), and $\gamma$-ray radiations. These emissions are produced by particles, which are (most likely) accelerated in the corona in a flaring loop. The accelerated electrons produce microwave and HXR emissions via synchrotron and bremsstrahlung processes (Hoyng et al. 1981; Sakao 1994; Kundu et al. 1994), while the interaction of accelerated protons and ions with the background ions produces $\gamma$-rays (Lin 1985). However, most of the energy of the particles goes into heating of the plasma via Coulomb collisions as they travel down to the footpoints. The heating and evaporation of the plasma produce secondary continuum and line emissions, from infrared to soft X-ray energy bands, which also carry information about the acceleration process. The purpose of the work presented here is to explore this second avenue of testing the acceleration models.

For the long run, our aim is to use the combined nonthermal and thermal signatures to distinguish between the different acceleration scenarios and constrain the characteristics of the specific acceleration models. This requires a combined treatment of the acceleration, transport, and radiation of particles and hydrodynamic (HD) response of the atmosphere to the energy input by particles. The first numerical study of particle transport in solar flares was carried out by Leach \& Petrosian (1981), who treated the transport and radiation by electrons using the Fokker-Planck transport equation taking into account the pitch-angle changes due to Coulomb collisions and magnetic field variations. This study was extended by McTiernan \& Petrosian (1990), who considered energy-loss and pitch-angle changes due to synchrotron emission. This transport-radiation code was later combined with an SA code (Park \& Petrosian 1995; Petrosian \& Donaghy 1999; Petrosian $\&$ Liu 2004) into the unified Stanford code (Petrosian et al. 2002).

The HD response has been investigated in several works (Mariska et al. 1989; Kašparová et al. 2009) by means of 1D 
numerical HD along a coronal loop. These works assumed that nonthermal electrons with a power-law spectrum were injected at the apex of the loop and used to approximate analytic expressions for the transport and energy deposition along the loop. Abbett \& Hawley (1999) and Allred et al. (2005) improved the results by including a detailed calculation of radiative transfer in the atmosphere.

We have embarked on development of more complete and self-consistent treatment of this important problem. Instead of using an ad hoc power law of injected electrons and approximate treatment of transport, we have combined the Stanford Fokker-Planck acceleration-transport code with HD codes, achieving a more accurate determination of the radiative signatures of flares. In our first paper, Liu et al. (2009, hereafter Paper I), we combined the Stanford code with the Naval Research Laboratory (NRL) hydro-code (Mariska et al. 1989), where we addressed some of the nonthermal aspects of the problem. Here we extend this study by taking into account the detailed calculation of radiative transfer in the atmosphere. Instead of the NRL code, we use the radiative HD (RADYN) code (Carlsson \& Stein 1992, 1997), in a modified version (Abbett \& Hawley 1999; Allred et al. 2005), and focus on intensities and shapes of several lines emitted at different heights. The main goal here is to show the effects of more realistic accelerated spectra of electrons as compared to those of power-law injected electrons. In a recent paper we have used the RADYN code to interpret spectroscopic observations of IR-optical-UV continuum and line emissions (Rubio da Costa et al. 2015). More such comparison with specific observations, both line and (thermal and nonthermal) continuum emissions, will be presented in future publications using this combined code.

This paper is organized as follows. In Section 2 we describe our model and the method used to solve the combined radiative HD and Fokker-Planck equations. In Section 3 we present results on plasma characteristics from the simulations and compare them with those from the power-law injection case. In Section 4 we focus on the effects of electron heating on the emission in the $\mathrm{H} \alpha$, Ca II K, He II $304 \AA$, and Si IV $1393 \AA$ lines. A brief summary and conclusions are given in Section 5. In Appendix A we present details of different energy terms and their influence on the atmospheric evolution, and in Appendix B we investigate the effects of different electron fluxes.

\section{MODEL AND METHOD}

\subsection{Assumptions and Geometry}

Magnetized plasma processes, in general, are commonly modeled with multidimensional magnetohydrodynamic (MHD) codes. However, in the solar corona the magnetic pressure dominates over the gas pressure (i.e., the plasma $\beta \ll 1$ ), so plasma flows mainly along magnetic field lines. This allows us to use the HD instead of MHD treatment of the problem.

We assume a one-dimensional, semicircular loop perpendicular to the solar surface with a constant diameter of $3 \mathrm{Mm}$. Adopting a symmetric boundary condition at the loop apex, we construct a computational domain containing a $10.4 \mathrm{Mm}$ long quarter circle, discretized in 191 grid points (a denser grid would be computationally too expensive). The loop extends in a plane-parallel model atmosphere from the corona to the bottom of the chromosphere at $z=0 \mathrm{Mm}$, where the optical depth $\tau_{5000}$ at a wavelength of $\lambda=5000 \AA$ is unity. The circular geometry is taken into account when calculating the $\mathrm{X}$-ray photoionization rates and the gravitational acceleration; otherwise, the loop is treated as a vertical cylinder.

For the preflare conditions we use the FP2 model of Abbett \& Hawley (1999), which was generated by adding a transition region and corona to the model atmosphere of Carlsson \& Stein (1997). The temperature is fixed to $10^{6} \mathrm{~K}$ at the loop apex. By running the code without external heating, the atmosphere relaxes to a state of HD equilibrium.

\subsection{Radiative Transfer and Hydrodynamics}

The RADYN code simultaneously solves the equations of HD, population conservation, and radiative transfer using a one-dimensional adaptive grid (Dorfi \& Drury 1987).

Atoms important to the chromospheric energy balance are treated in non-local thermodynamical equilibrium (NLTE). These include a six-level plus continuum hydrogen atom; a sixlevel plus continuum, singly ionized calcium atom; a nine-level plus continuum helium atom; and a four-level plus continuum, singly ionized magnesium atom. The transitions that are treated in detail are given in Table 1 of Abbett \& Hawley (1999). Complete redistribution (CRD) is assumed for all lines, except for the Lyman series, in which partial frequency redistribution is mimicked by truncating the profiles at 10 Doppler widths (Milkey \& Mihalas 1973). Other atomic species are included in the calculation as background continua in LTE, using the Uppsala opacity package of Gustafsson (1973).

As described in Allred et al. (2005), the radiative HD code includes the calculation of photoionization heating resulting from high-temperature, soft-X-ray-emitting regions, as well as the calculation of optically thin cooling due to thermal bremsstrahlung and collisionally excited metal transitions. An adjustment in the calculation of the conductive flux has also been taken into account in order to avoid unphysical large values in the transition region-where temperature gradients are large. HD effects due to gravity, thermal conduction, and compressional viscosity are considered as described in Abbett \& Hawley (1999).

Inclusion of the radiative transfer calculation gives us the advantage, with respect to Paper I, to investigate how the electron deposition in the chromosphere affects the emission lines originating from several heights (see Section 4).

\subsection{Electron Acceleration, Transport, and Energy Deposition}

FLARE, the Stanford unified acceleration-transport code (McTiernan \& Petrosian 1990; Petrosian \& Liu 2004), consists of two modules. The acceleration module calculates the spectrum of the electrons accelerated stochastically by turbulence in the acceleration region (assumed to be located at the loop top; for observational evidence see, e.g., Liu et al. 2013) and the spectrum of electrons escaping down to the footpoints. The transport module then calculates the evolution of the spectrum and pitch-angle distribution along the loop of the escaping electrons from the acceleration site. The code includes energy and pitch-angle diffusion due to Coulomb collisions and synchrotron radiation.

One of the main inputs to the RADYN code is the heating rate $Q_{e}(s)$ (in units of $\mathrm{erg} \mathrm{s}^{-1} \mathrm{~cm}^{-3}$ ) due to accelerated electrons as a function of position $s$ along the loop, which is included as a source of external heating in the energy conservation equation 
Table 1

Summary of the Different Simulation Runs and the Atmospheric Parameters for Different Flaring Conditions

\begin{tabular}{|c|c|c|c|c|c|c|c|c|}
\hline Run & Injected $e^{-}$Distribution & $\underset{\left(\mathrm{erg} \mathrm{s}^{-1} \mathrm{~cm}^{-2}\right)}{\mathfrak{F}_{\max }}$ & $\begin{array}{c}v_{\max } \\
\left(\mathrm{km} \mathrm{s}^{-1}\right)\end{array}$ & $\begin{array}{c}t\left(v_{\max }\right) \\
(\mathrm{s})\end{array}$ & $\begin{array}{c}v_{\min } \\
\left(\mathrm{km} \mathrm{s}^{-1}\right)\end{array}$ & $\begin{array}{c}t\left(v_{\min }\right) \\
(\mathrm{s})\end{array}$ & $\begin{array}{c}T_{\max } \\
(\mathrm{K})\end{array}$ & $\begin{array}{l}t\left(T_{\max }\right) \\
\quad(\mathrm{s})\end{array}$ \\
\hline$\overline{\mathrm{PL}}$ & $\delta=5 ; E_{\mathrm{c}}=15 \mathrm{keV}$ & $1.2 \times 10^{10}$ & 483 & 46 & -44 & 30 & $9.5 \times 10^{6}$ & 75 \\
\hline SA1 & Stochastic acceleration & $1.2 \times 10^{10}$ & 750 & 32 & -41 & 10 & $2.3 \times 10^{7}$ & 60 \\
\hline SA3 & Stochastic acceleration & $5.7 \times 10^{8}$ & 377 & 77 & -28 & 31 & $8.0 \times 10^{6}$ & 61 \\
\hline
\end{tabular}

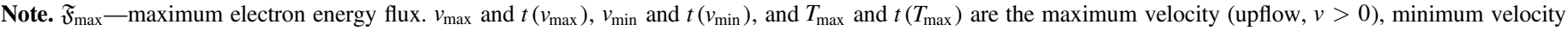
(downflow, $v<0$ ), and maximum temperature and their corresponding times, respectively.

(see Equation (2) in Appendix A). Abbett \& Hawley (1999) assumed a beam of electrons with a power-law spectrum with an index $\delta$ and low-energy cutoff $E_{\mathrm{c}}$ and used the approximate analytic expression of Emslie (1981) to calculate the heating rate. Here we use the electron spectrum from the accelerationtransport code, which in general includes a quasi-thermal plus a nonthermal component. We then calculate the heating rate $Q_{e}(s)$ using the spatial variation of the spectrum and pitch-angle distribution $f(E, \mu, s)$ of the electrons, following the procedure described in Section 3.1 of Paper I.

\subsection{Combining FLARE and RADYN}

We followed the approach detailed in Paper I to combine the FLARE and RADYN codes. In brief, we note that the current particle transport module of FLARE can provide only a steadystate solution. To perform time-dependent simulation, RADYN calls the transport module every $\Delta t=1 \mathrm{~s}$. This interval of communication between the two codes allows for a steady-state solution of particle kinetics, whose timescales are orders of magnitude shorter than HD timescales. This interval also provides a trade-off for computational efficiency.

In each iteration, the RADYN code passes the electron density of the atmosphere as a function of distance from the top of the loop $\left(n_{e}(s)\right)$ to the FLARE code. The FLARE code calculates the electron heating rate $Q_{e}(N)$ as a function of column density $N_{e}=\int_{0}^{s} n_{e}(s) d s$ and returns it to the RADYN code, which then simulates the evolution of the atmosphere and advances in time for $\Delta t=1 \mathrm{~s}$. Within this interval, we assume that the accelerated electron flux remains constant and so does $Q_{e}\left(N_{e}\right)$. Therefore, the temporal evolution of the heating rate $Q_{e}(s, t)$ as a function of distance solely depends on the spatial redistribution of the electron density $n_{e}(s, t)$ with time, as described in Section 3.2 of Paper I.

By including the radiative transfer calculation, one of the advantages over Paper $\mathrm{I}$ is the detailed study of how the electron deposition in the chromosphere affects the emission of the lines at several heights (see Section 4).

\subsection{Simulation Runs}

We have performed four simulation runs with different injected electron spectra, as summarized in Table 1, to investigate their different atmospheric response. These runs were carefully designed and allowed us to compare our results using the acceleration-model-based spectra with those carried out in the past using a simple power law. In the Run PL, we use a single power-law electron spectrum of index $\delta=5$ and lowenergy cutoff of $E_{\mathrm{c}}=15 \mathrm{keV}$ and the analytic expression of Emslie $(1978,1981)$ to calculate the electron heating rate $Q_{e}$. Runs SA1-SA3 use the result of the SA model for the injected

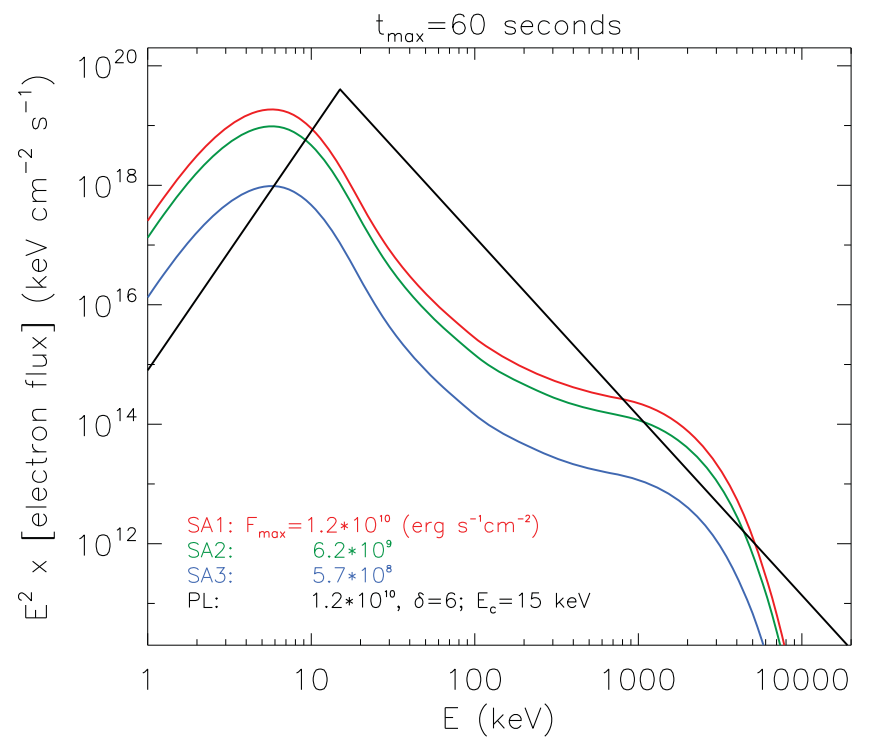

Figure 1. Spectra of angle-integrated electron fluxes, $F(E)$, times $E^{2}$ at the loop top and $t_{\max }=60 \mathrm{~s}$ for different simulation runs labeled with the corresponding energy fluxes $\mathfrak{F}_{\max }$. The black line represents a power law with a spectral index $\delta=5$ and low-energy cutoff $E_{\mathrm{c}}=15 \mathrm{keV}$ (Run PL), while the colored lines represent stochastically accelerated electron spectra (Runs SA1-SA3).

electron spectra, for which we prescribed a common characteristic acceleration timescale of $\tau_{p}=1 / 70 \mathrm{~s}$ (Petrosian \& Liu 2004) and thus the same spectral shape. For the SA runs we calculate the $Q_{e}$ using the approach described in Section 2.3. Note that Run SA1 has identical electron spectra with Run N in Paper I and Run PL has a lower spectral index than Run O in Paper I. Each simulation lasts $120 \mathrm{~s}$, with the electron energy flux $\mathfrak{F}(t)$ increasing linearly until $t_{\max }=60 \mathrm{~s}$ up to $\mathfrak{F}_{\max }$ and then decreasing for another $60 \mathrm{~s}$. In all the runs we keep the electron spectral shape constant in time.

Figure 1 compares the spectra of angle-integrated electron flux at the top of the loop for the different runs shown in Table 1. In the spectral range approximated for HXR bremsstrahlung emission, the slope of the PL spectrum has been chosen to be similar to the one of the SA Run. The main difference between the two models is the absence of the quasithermal electrons in the PL model, and the secondary difference is the rollover in the SA model at highest energies.

We should, however, note that these differences are not unique to the SA model. Most models, when accelerating particles from a thermal pool, produce a hotter quasi-thermal component with a power-law tail because of Coulomb collision effects. Interested readers are referred to, for example, Petrosian \& East (2008) for a generic acceleration model and 


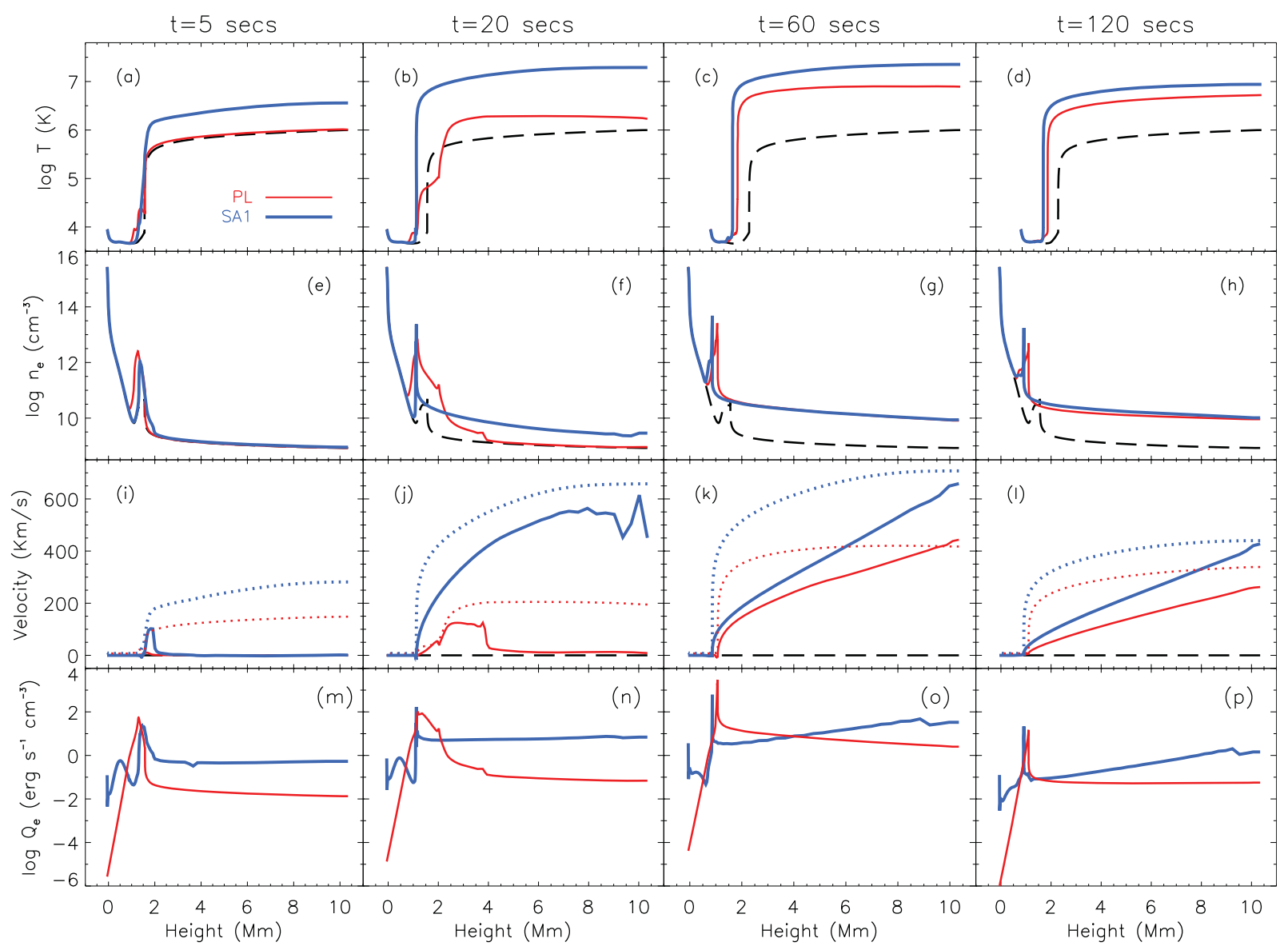

Figure 2. Variation with distance along the loop of the atmospheric parameters (temperature, electron density, velocity of the plasma, and electron heating rate per unit volume $\left[Q_{e}\right]$, where positive velocities refer to upflows and negative velocities to downflows) in the PL (red line) and SA1 (blue thick line) models. The black dashed lines show the initial values, and the dotted lines in the bottom panels represent the sound speed. The columns are for $t=5,20,60$, and $120 \mathrm{~s}$.

(An animation of this figure is available.)

Sironi \& Spitkovsky (2009) for shock acceleration using particle-in-cell simulations. We also note that the selection of the spectral parameters has not been done to study any particular flare.

In the next section we investigate how the atmosphere responds to the injection of single power-law electrons (Run PL) and stochastically accelerated electrons (Run SA1), and in Appendix B we compare results of Runs SA1, SA2, and SA3 to investigate how the atmosphere responds to the variation of the electron flux.

\section{COMPARISON OF SINGLE POWER-LAW INJECTION WITH STOCHASTIC ACCELERATION}

In order to investigate how the acceleration and transport of electrons affects the atmospheric response, we compare the results of injection of single power-law electrons (Run PL) and of stochastically accelerated electrons (Run SA1), which have the same total energy flux at any moment.

\subsection{Atmospheric Evolution}

Figure 2 and the online movie shows the temporal evolution of the atmosphere. In general, SA1 has higher values than PL for all the atmospheric variables (see Table 1), qualitatively consistent with those of Runs $\mathrm{N}$ and $\mathrm{O}$ in Paper I, respectively. For example, the corona is heated more rapidly to higher temperatures in SA1 than that in PL. The maximum coronal temperature of $2.3 \times 10^{7} \mathrm{~K}$ is reached at $t=60 \mathrm{~s}$ in SA, compared with a lower value of $9.5 \times 10^{6} \mathrm{~K}$ at $74 \mathrm{~s}$ in PL (Figure 3(c)). As detailed in Section 3.2, such contrasts are due to the different spatial distributions of collisional heating by nonthermal electrons of the two models, even though they have the same energy input to the loop as a whole. This factor of 2.4 difference in the maximum coronal temperature is much greater than the factor of 1.2 difference between similar runs in Paper I (see their Table 1), which points to the importance of detailed radiative transfer calculation included here.

At early times, electron heating in the transition region and chromosphere causes an overpressure that drives both upflows (i.e., chromospheric evaporation) and downflows of plasma, with higher speeds in the case of SA1. At $t=5 \mathrm{~s}$, for example, SA1 has the maximum upflow and downflow velocities of 102 and $-7 \mathrm{~km} \mathrm{~s}^{-1}$, respectively, compared with 13 and $-0.4 \mathrm{~km} \mathrm{~s}^{-1}$ in PL (see Figure 3 for the temporal evolution). As a result of the upflow, part of the initial transition region and chromospheric material is converted into coronal mass, and the new transition region recedes to lower heights between $z=0.99$ and $1.10 \mathrm{Mm}$.

At $t=5 \mathrm{~s}$ most of the energy is deposited in a narrow region located in the upper chromosphere at a height of $z=1.27 \mathrm{Mm}$ for PL and at $1.34 \mathrm{Mm}$ for SA1. The nonthermal electrons quickly heat this region to temperatures greater than $10^{4} \mathrm{~K}$. The 


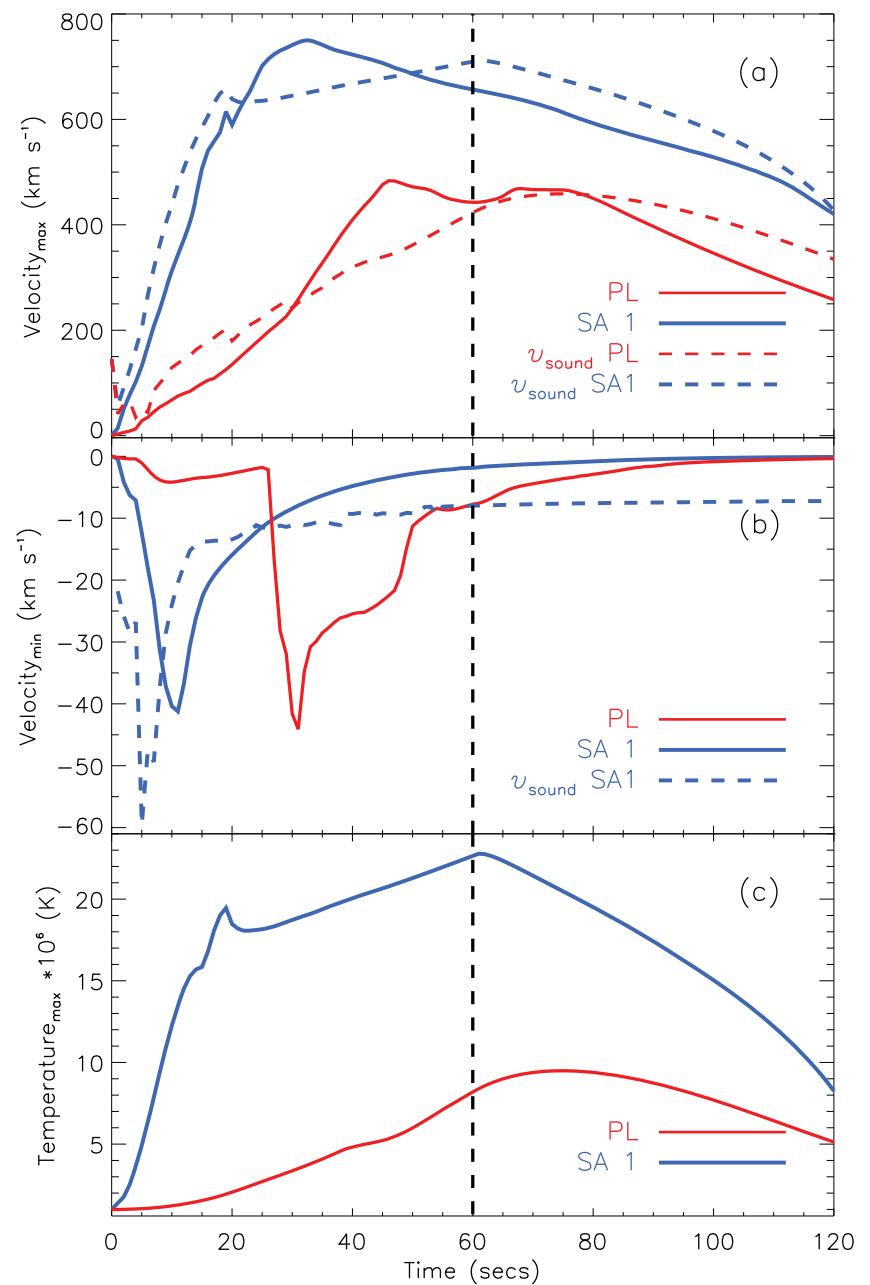

Figure 3. Temporal evolution of the maximum (a) upflow velocity, (b) downflow velocity, and (c) temperature within the loop for Runs PL (red line) and SA1 (blue thick line). The dashed curve indicates the sound speed at the corresponding location of the maximum (a) upflow and (b) downflow velocity for each run.

location of the maximum nonthermal energy deposition gradually moves downward, reaching a height of $1.17 \mathrm{Mm}$ for PL and $1.12 \mathrm{Mm}$ for SA1 at $t=20 \mathrm{~s}$. A more detailed discussion on how the electron heating rate affects the atmosphere is given in Appendix A, where the height distribution of the different energy terms and their temporal evolution are presented. We note that in the case of PL a significant fraction of hydrogen is ionized at $t=5 \mathrm{~s}$ and $z=1.18 \mathrm{Mm}$ and thus the energy can no longer be effectively radiated away by bound-bound transitions.

Figure 3(a) shows the temporal evolution of the maximum velocity along the loop (solid lines) and the sound velocity at the same position (dashed lines). Again, SA1 has higher plasma velocities, creating an HD shock with supersonic speeds between $t=22$ and $49 \mathrm{~s}$, while in the PL case, a shock is developed at later times between $t=30$ and $78 \mathrm{~s}$. For SA1, the maximum upflow velocity of $v_{\max }=750 \mathrm{~km} \mathrm{~s}^{-1}$ with a Mach number of 1.15 is attained at $t=32 \mathrm{~s}$, while for PL the maximum velocity $v_{\max }=483 \mathrm{~km} \mathrm{~s}^{-1}$ with a Mach number of 1.41 occurs at $t=46 \mathrm{~s}$.

As shown in Figure 3(b), between $t=9$ and $24 \mathrm{~s}$ for the SA1 Run, the downflow plasma velocity exceeds the sound speed and forms a downward-propagating shock. In contrast, the sound speed (not shown) for the PL case in panel (b) is alway greater than the maximum downflow speed, except for a brief period of $3 \mathrm{~s}$ from $t=32$ to $t=34 \mathrm{~s}$.

In Figure 3(c) we can also see that the temporal evolution of the maximum temperature along the loop follows a similar general trend to the electron flux $\mathfrak{F}(t)$, but the temperature in the SA1 run increases much faster than that in the PL run.

Figure 4 shows the temperature distribution of the plasma velocity at different times. The two runs exhibit distinct behaviors at early times. At $t=5$ and $20 \mathrm{~s}$ (panels (a) and (b)), for example, in the PL case, mainly low-speed upflows in the $10^{4}-10^{6} \mathrm{~K}$ range are present. In the SA1 case, downflows occur at low temperatures and high-speed upflows at high temperatures, with their division temperature increasing with time from a few times $10^{4} \mathrm{~K}$ to nearly $10^{6} \mathrm{~K}$. Comparably slower downflows take place in the PL case only at later times (e.g., $60 \mathrm{~s}$ ). As time progresses toward the late phase of the flare, such a distinction diminishes to lesser degrees, with mainly upflows present in both cases whose speed rapidly grows with temperature.

Such distinct temperature distributions of the plasma velocity, especially early during a flare, are manifested in the Doppler shifts of emission lines shown in Figures 6, 9, 12, and 14, which will be discussed later, and demonstrate their sensitive dependence on the spectra of accelerated electrons. When compared with observations, such distributions can be used as diagnostics to constrain particle acceleration mechanisms. For example, Milligan \& Dennis (2009) used the EUV Imaging Spectrometer (EIS) on board Hinode to measure the Doppler velocities of emission lines at formation temperatures ranging from 0.05 to $16 \mathrm{MK}$. They found a temperature distribution of Doppler velocity akin to that of SA1 shown in Figure 4(b). Specifically, their -60 to $-30 \mathrm{~km} \mathrm{~s}^{-1}$ downflow speeds within $0.6-1.5 \mathrm{MK}$ are comparable to those in our SA1 case at $t=9-12 \mathrm{~s}$. They found a slightly higher division temperature of $\sim 2 \mathrm{MK}$ between up- and downflows. This minor difference could be partly due to the greater electron energy flux of $5 \times 10^{10} \mathrm{erg} \mathrm{cm}^{-2} \mathrm{~s}^{-1}$ estimated in their C-class flare, about five times that adopted in our simulation.

\subsection{Evolution of the Electron Heating Rate}

The height distribution of the electron heating rate $Q_{e}(s)$ is shown in the bottom row of Figure 2 at $t=5,20,60$, and $120 \mathrm{~s}$ for PL (red line) and SA1 (blue thick line). In general, both runs present a peak in the upper chromosphere. However, $Q_{e}$ in $\mathrm{PL}$ is higher in the chromosphere but lower in the corona than that in SA1.

In PL the electron energy is mostly deposited in the upper chromosphere (at $z=1.18 \mathrm{Mm}$ for $t=1 \mathrm{~s}$ ). The electrons interact with the plasma, ionizing hydrogen and helium. A step in the heating rate appears at the chromospheric evaporation front owing to the density jump (e.g., at $z \approx 3.7 \mathrm{Mm}$ in Figure 2(n)). During the decay phase of the flare, the heating rate decreases throughout the loop, but there is more fractional reduction in the chromosphere than in the corona.

In the case of $\mathrm{SA} 1, Q_{e}$ is mostly concentrated around $z \approx$ $1.45 \mathrm{Mm}$ after $t=1 \mathrm{~s}$, covering a broader region than in PL. This is because the stochastically accelerated electrons have a quasi-thermal spectral component plus a nonthermal tail covering a broader energy range, as shown in Figure 1. The quasi-thermal component carries considerable energy contents and causes significant heating in the corona that reaches 

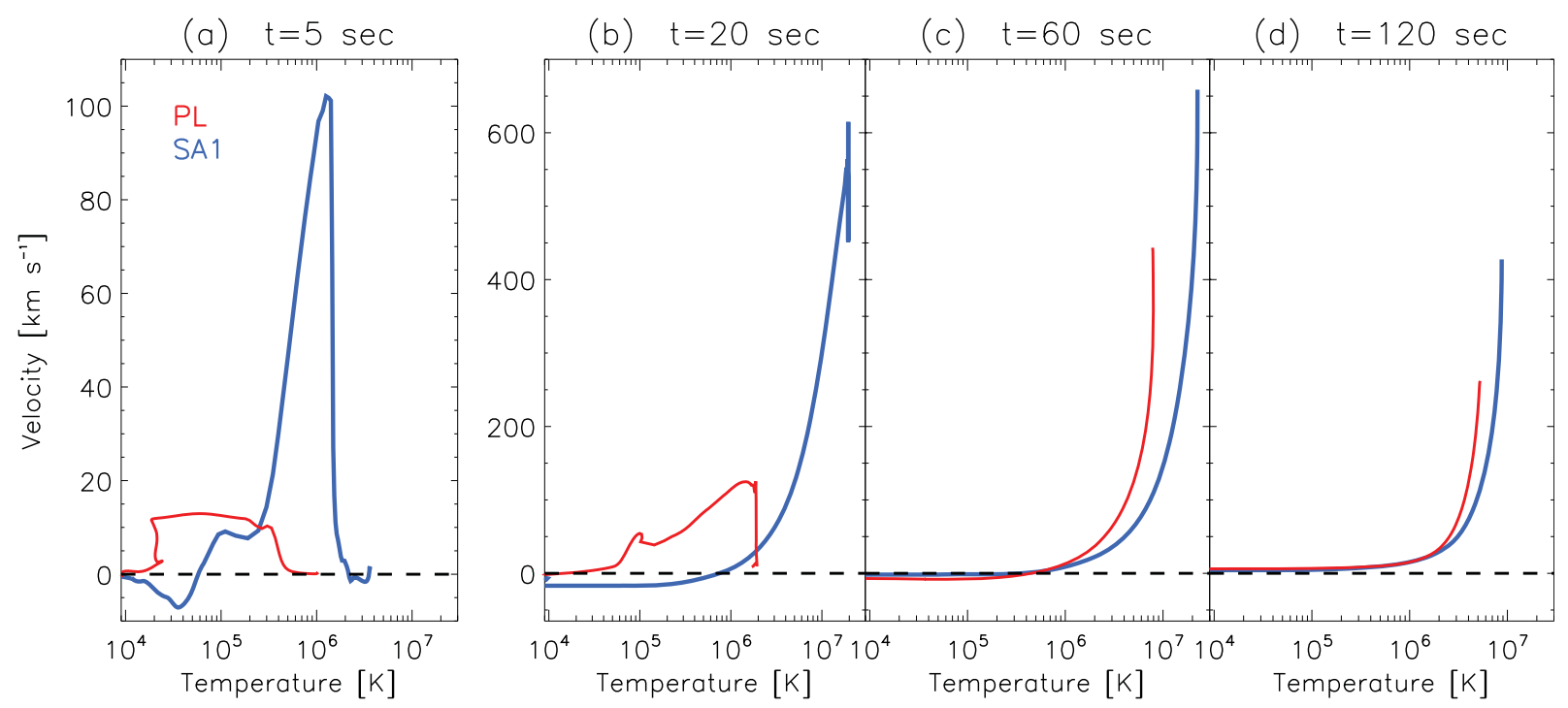

Figure 4. Temporal evolution of the velocity vs. temperature for PL (red solid line) and SA1 (blue solid thick line).
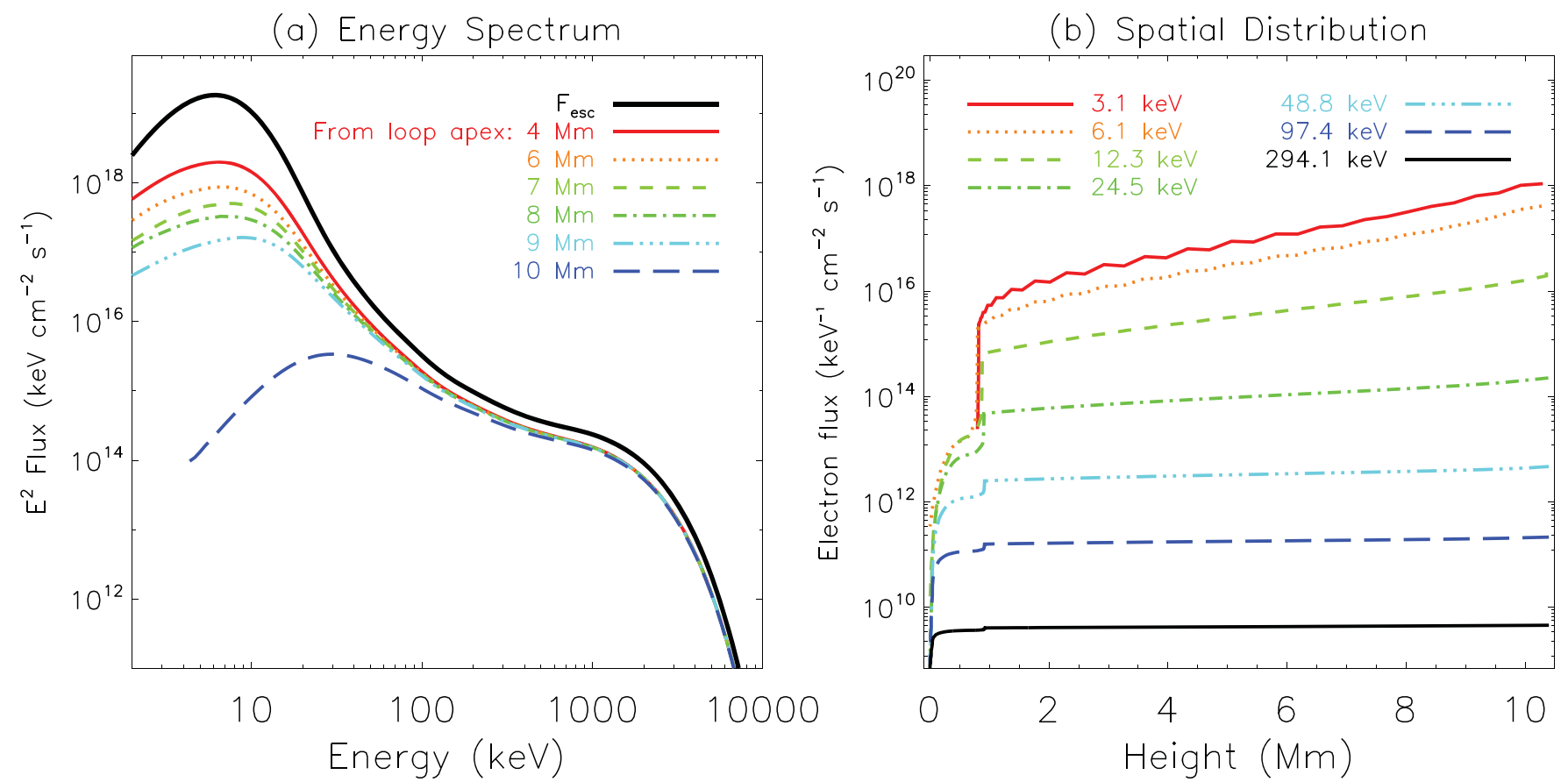

Figure 5. Energy and spatial distributions of nonthermal electrons at $t_{\max }=60 \mathrm{~s}$ in Run SA1. (a) Energy spectra of angle-integrated electron flux multiplied by the energy squared. The solid black line is the flux $\left(F_{\text {esc }}\right)$ of electrons escaping the acceleration region at the loop apex that serves as the injection to the transport portion of the loop. The color-coded curves (from red to blue) of different line styles represent fluxes at growing distances of 4, 6, 7, 8, 9, and 10 Mm from the loop apex. (b) Spatial distribution of angle-integrated electron flux as a function of distance along the loop. The color-coded lines represent fluxes at different electron energies, 3 , $6.1,12.3,24.5,48.8,97.4$, and $294.1 \mathrm{keV}$. Note the large step at the transition region, especially at low energies.

temperatures of $11 \mathrm{MK}$ at $t=10 \mathrm{~s}$ and $23 \mathrm{MK}$ at $60 \mathrm{~s}$. The secondary heating peak in the lower chromosphere is due to the nonthermal tail that exceeds the power-law spectrum from $\approx 800 \mathrm{keV}$ to $6 \mathrm{MeV}$. The sharp spike in the photosphere is due to the increase of the electron density at that height.

The above behaviors of the electron heating rate can be better understood by examining the energy and spatial distributions of the nonthermal electrons. Figure 5(a) shows the energy spectra of $E^{2} F(E, s)$ at selected distances $s$ from the top of the loop for Run SA1 at $t=60 \mathrm{~s}$, where $F(E, s)=\int_{-1}^{1} v f(E, \mu, s) d \mu$ is the pitch-angle-integrated electron flux. As expected, low-energy electrons suffer more losses at higher altitudes than the high-energy electrons. This is mainly because of the $1 / v$ dependence of the Coulomb collision energy-loss rate. Note that the large decrease of electron flux from $s=9$ to $10 \mathrm{Mm}$ results from the change of location from above to below the transition region with a sudden increase of the atmospheric density.

Figure 5(b) shows the spatial distribution of the electron flux $F(E, z)$ along the loop at selected electron energies for Run SA1 at $t=60 \mathrm{~s}$ corresponding to Figure 5(a). In general, the electron flux decreases with distance from the injection site at $z=10.40 \mathrm{Mm}$. The slope $(d F(E, z) / d z)$ is steeper at lower 


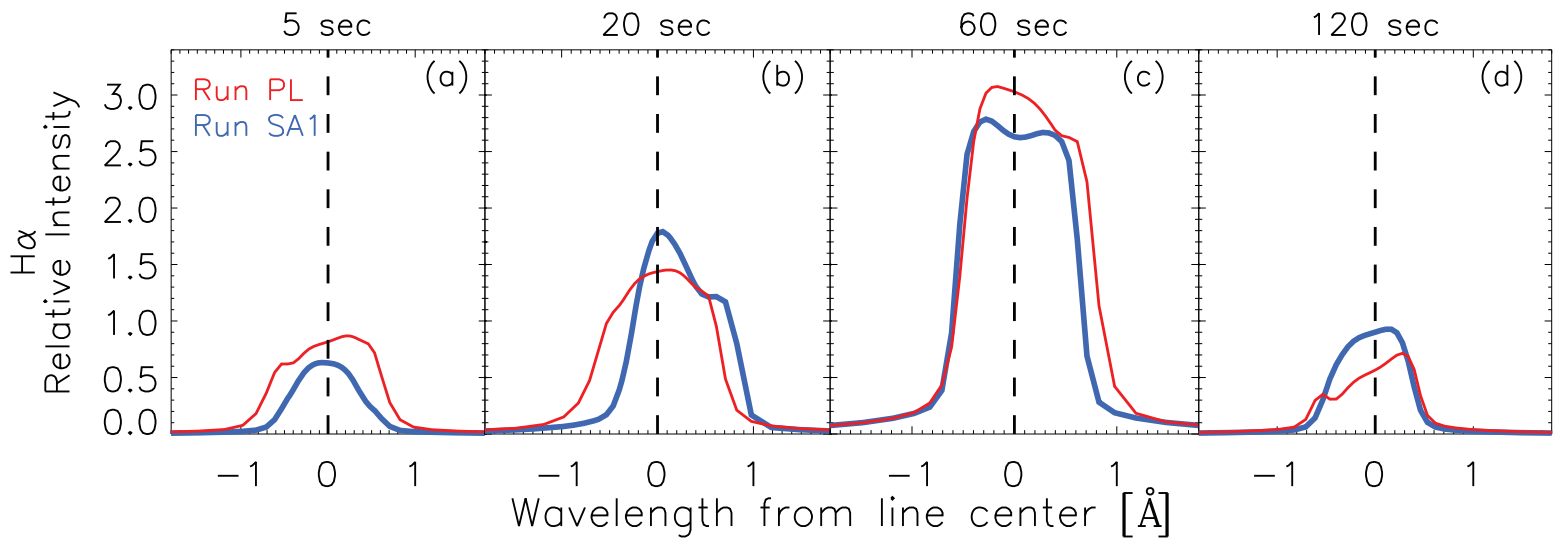

Figure 6. H $\alpha 6563 \AA$ line profiles at $t=5,20,60$, and $120 \mathrm{~s}$ for runs PL (red line) and SA1 (blue thick line). The emission at the initial time has been subtracted, and the intensity has been normalized to the continuum. The vertical dashed line indicates the line-center rest wavelength.

energies because low-energy electrons lose energy faster than high-energy electrons. This slope also depends on the ambient density $n_{e}$, because $d F(E, z) / d z=n_{e}[d F(E, N) / d N]$, where $d F(E, N) / d N$ is generally a smooth function of column density $N$ (McTiernan \& Petrosian 1990). As such, a sharp drop in the electron flux, more prominent at lower energies, occurs at the transition region $(z=0.8 \mathrm{Mm})$ owing to the sharp ambient density rise. In addition, the slope is proportional to the electron heating rate (see Equations (10) and (11) in Paper I) and thus accounts for its shape, especially the narrow peak at the transition region, as shown in Figures $2(\mathrm{~m})-(\mathrm{p})$.

\section{LINE EMISSION}

One of the advantages of this work over previous studies (e.g., Liu et al. 2009) is the inclusion of the detailed radiative transfer calculation of emission lines treated in NLTE and thus the capability of synthesizing line emission from simulation results at different height formations with the aim of studying how the energy deposition affects the lower atmosphere. In this section we will examine four optically thick emission lines, H $\alpha$, Ca II K, He II $304 \AA$ and Si IV $1393 \AA$, which are common observables and cover formation heights from the upper photosphere to the transition region. For example, the $\mathrm{H} \alpha$ and $\mathrm{Ca}$ II $\mathrm{K}$ lines are routinely observed by ground-based facilities, e.g., at the Big Bear Solar Observatory (Johannesson et al. 1995, 1998) and the Kanzelhöhe Observatory (Pötzi et al. 2013). The Extreme-ultraviolet Variability Experiment on board the Solar Dynamics Observatory (Woods et al. 2012) covers the He II $304 \AA$ and Si IV $1393 \AA$ lines; the latter is also covered by the Interface Region Imaging Spectrograph (IRIS; De Pontieu et al. 2014). We intend to perform detailed comparison with observations in future investigations.

In order to better understand the behavior of these lines, we write the formal solution of the radiative transfer equation for emergent intensity:

$$
I_{\nu}^{0}=\frac{1}{\mu} \int_{z} S_{\nu} e^{\frac{-\tau_{\nu}}{\mu}} \chi_{\nu} d z=\frac{1}{\mu} \int_{z} C_{i} d z
$$

where $S_{\nu}$ is the source function, which is defined as the ratio between the emissivity and the opacity of the atmosphere; $\tau_{\nu}$ is the monochromatic optical depth; and the integrand $C_{i}$ is the so-called intensity contribution function, which represents the intensity emanating from height $z$.

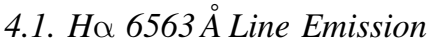

The $\mathrm{H} \alpha$ line is one of the most commonly observed lines that allow us to study the chromospheric response during a solar flare. It is sensitive to the flux of nonthermal electrons precipitating to the chromosphere (Švestka 1976; Kašparová et al. 2009; Rubio da Costa et al. 2015) and a complex line formed in a broad height range, from the upper photosphere to the lower chromosphere, making its interpretation nontrivial.

Figure 6 shows the temporal evolution of the resulting excess $\mathrm{H} \alpha$ line profile, where the quiet-Sun emission at $t=0 \mathrm{~s}$ has been subtracted to emphasize the changes during the flare and the differences between Runs PL (red line) and SA1 (blue line). In both runs, the line core presents a flattening due to the sudden behavior change of the source function in a very thin atmospheric layer, as previously reported by Rubio da Costa et al. (2015). The line is broader and the intensity at the core is stronger for PL most of the time, while SA1 shows stronger asymmetry (mostly blueshifts associated with upflows) due to the higher velocities in the chromosphere. Close to $t_{\max }=60 \mathrm{~s}$ the profile presents a stronger blueshifted peak in the line center and redshifts in the wings, indicating plasma upflows (evaporation) from the chromosphere to the transition region and downflows in the chromosphere.

The above behavior can be better understood by examining the contribution function, as shown in Figure 7. We find that the photons in the wings of the line originate from the low chromosphere (at $z=0.12 \mathrm{Mm}$ ), where the plasma velocity is almost zero and almost constant in time. Therefore, the wing emission changes little in time and is very similar in both runs. In contrast, the line core is formed in the transition region, whose height changes in time and where the plasma velocity suddenly increases as a result of upflows. For SA1, this region is situated at a lower height, and thus the overall height range of $\mathrm{H} \alpha$ line formation is narrower than that of PL. The PL model has lower velocities and covers a broader formation height range at the line core. Moreover, the plasma temperature and density play an important role in the formation of the line and might explain the differences in the core emission between the two runs (Leenaarts et al. 2012).

Integrating the intensity along the $\mathrm{H} \alpha$ line profile for a width of $\Delta \lambda=3.8 \AA$ yields the light curve shown in Figure 8(a). As can be seen, for SA1 the $\mathrm{H} \alpha$ emission is directly correlated with the evolution of the flux of electrons, peaking only $4 \mathrm{~s}$ after $t_{\text {max }}$. However, for PL, the emission exhibits a plateau after $47 \mathrm{~s}$, peaking exactly at $t_{\max }$. 


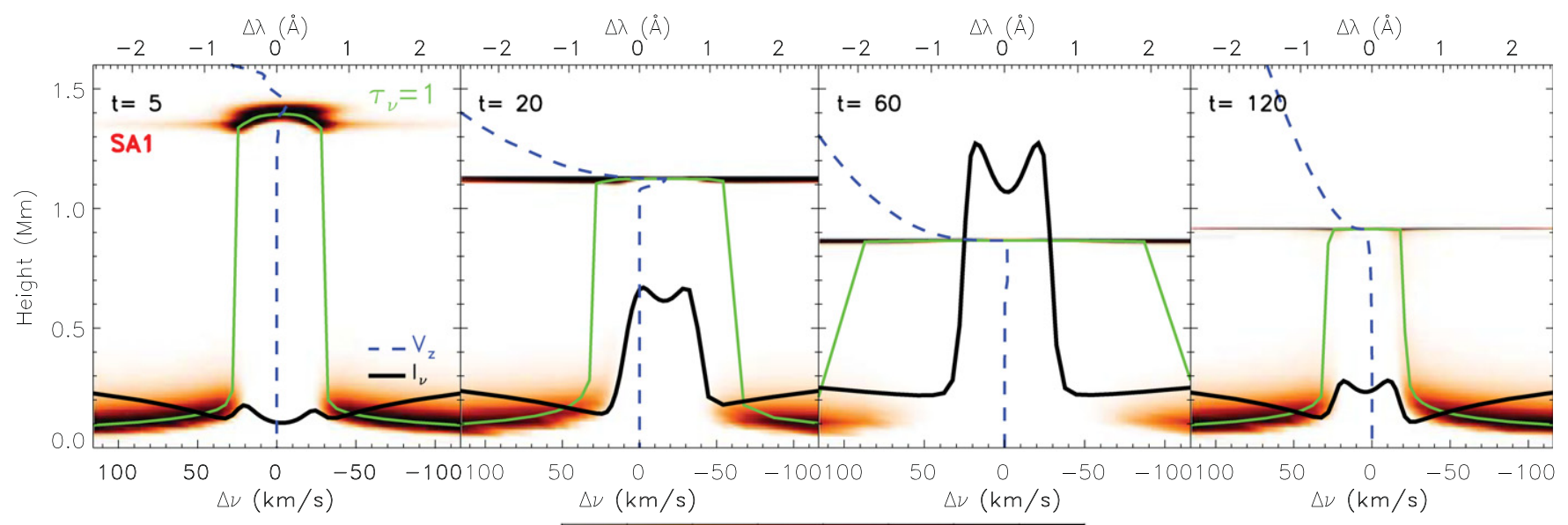

$5.0 \times 10^{-13} 1.0 \times 10^{-12} \quad 1.5 \times 10^{-12} \quad 2.0 \times 10^{-12}$

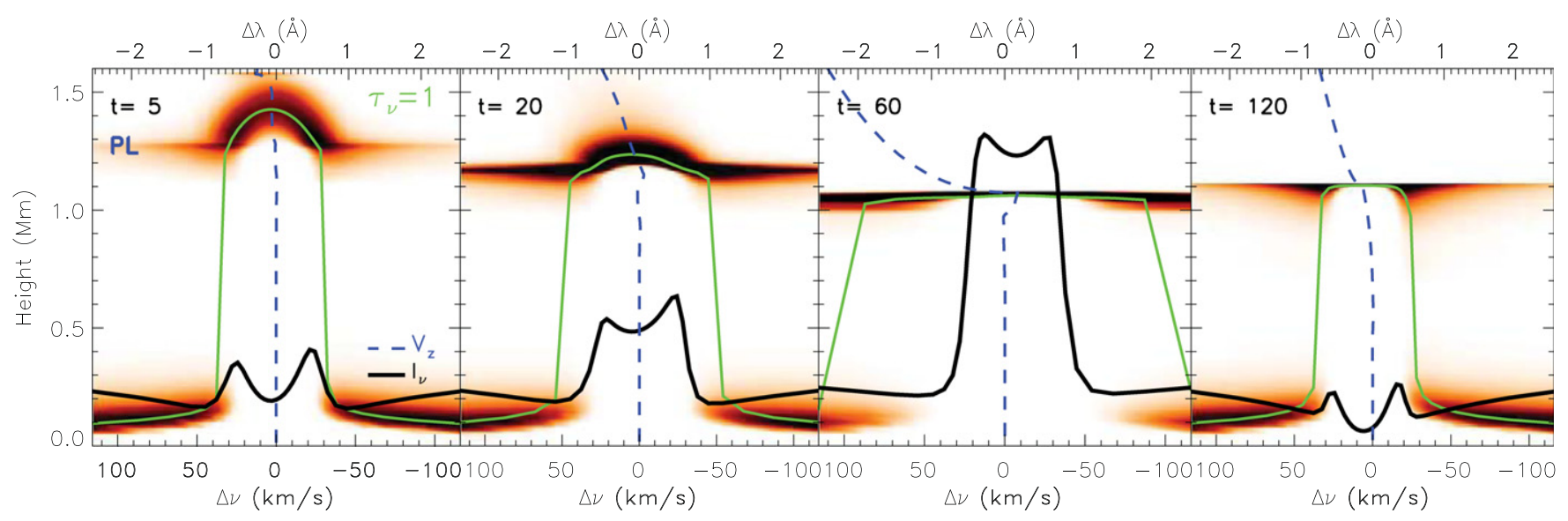

Figure 7. Intensity contribution function, as defined in Equation (1), for $\mathrm{H} \alpha$ as a function of frequency (bottom axis) and height (vertical axes), shown as reversed color maps (darker for higher values). The frequencies are in velocity units such that positive and negative velocities represent upflows and downflows of plasma, respectively. The dashed blue line represents the atmospheric velocity stratification; the solid green line shows the height at which $\tau_{\nu}=1$, and the black thick line shows the line profile (including the quiet-Sun emission). Top panel: SA1 Run; bottom panel: PL Run. All panels have the same color scale as indicated on the color bar.

\subsection{He II $304 \AA$ Line Emission}

The He II $304 \AA$ Alne is an optically thick line associated with the transition $1 s{ }^{2} S_{1 / 2}-2 p{ }^{2} P_{2}^{0}$. Under ionization equilibrium conditions, in general, it is formed in a narrow layer in the transition region, corresponding to temperatures of $\approx 5-8 \times 10^{4} \mathrm{~K}$ (O'Dwyer et al. 2010). However, Golding et al. (2014) noted that the details of its formation are still not well understood. Zirin (1988) showed that the photoionization and recombination processes play an important role in the He II $304 \AA$ Åmission. Jordan (1975) (see also Andretta et al. 2003) found that the observed intensity of the helium lines is higher than that estimated from observations of other EUV lines. They noted that the inclusion of high-energy electrons and cold ions in the models enhances the synthetic intensities. Based on this idea, Laming \& Feldman (1992) modeled the He I and He II emission during the impulsive phase of a flare by including the effects of high-energy electrons.

In Figure 9 we note large differences between PL and SA1, indicating that the $\mathrm{He}$ II $304 \AA$ emission is very sensitive to the spectra of the nonthermal electrons. In fact, the intensity in PL changes by two orders of magnitude within $9 \mathrm{~s}$ of the simulation. Whenever there are large velocity variations in the formation region, the line profile shows strong asymmetries. In particular, the line profile of the PL model at $t=20 \mathrm{~s}$ shows a strong blueshift due to the evaporation of material to upper layers.

Studying the monochromatic optical depth (green line in Figure 10), we find that the photons of the He II $304 \AA$ line are emitted in a very narrow region situated at the bottom of the transition region. The height range of the formation region varies between 5 and $300 \mathrm{~km}$ for the PL model, being even narrower (between 2 and $5 \mathrm{~km}$ after $t=10 \mathrm{~s}$ ) for the SA1 model. Figure 11 shows the He III ionization fraction as a function of height and temperature. It indicates that the He II atoms become completely ionized within a narrow height range in the transition region. At $t=20 \mathrm{~s}$, the PL model presents a gradual change of the ionization fraction over a broad height range, which is related to the broad formation heights shown in Figure 10 at this time.

From the intensity contribution function of Figure 10 we find that most of the intensity comes from $\approx \pm 0.02 \AA$ from the line center, and thus the line center appears as a dip. The line profile is very sensitive to plasma velocity changes as shown, e.g., at $t=20 \mathrm{~s}$ for the PL model. The temperature range at the formation height is of the order of (1-2) $\times 10^{5} \mathrm{~K}$ (see the pink symbols in Figure 11), more than twice as high as the expected values from O'Dwyer et al. (2010) and consistent with the simulation results reported by Golding et al. (2014). In addition, Jordan et al. (1993) found that the He II 


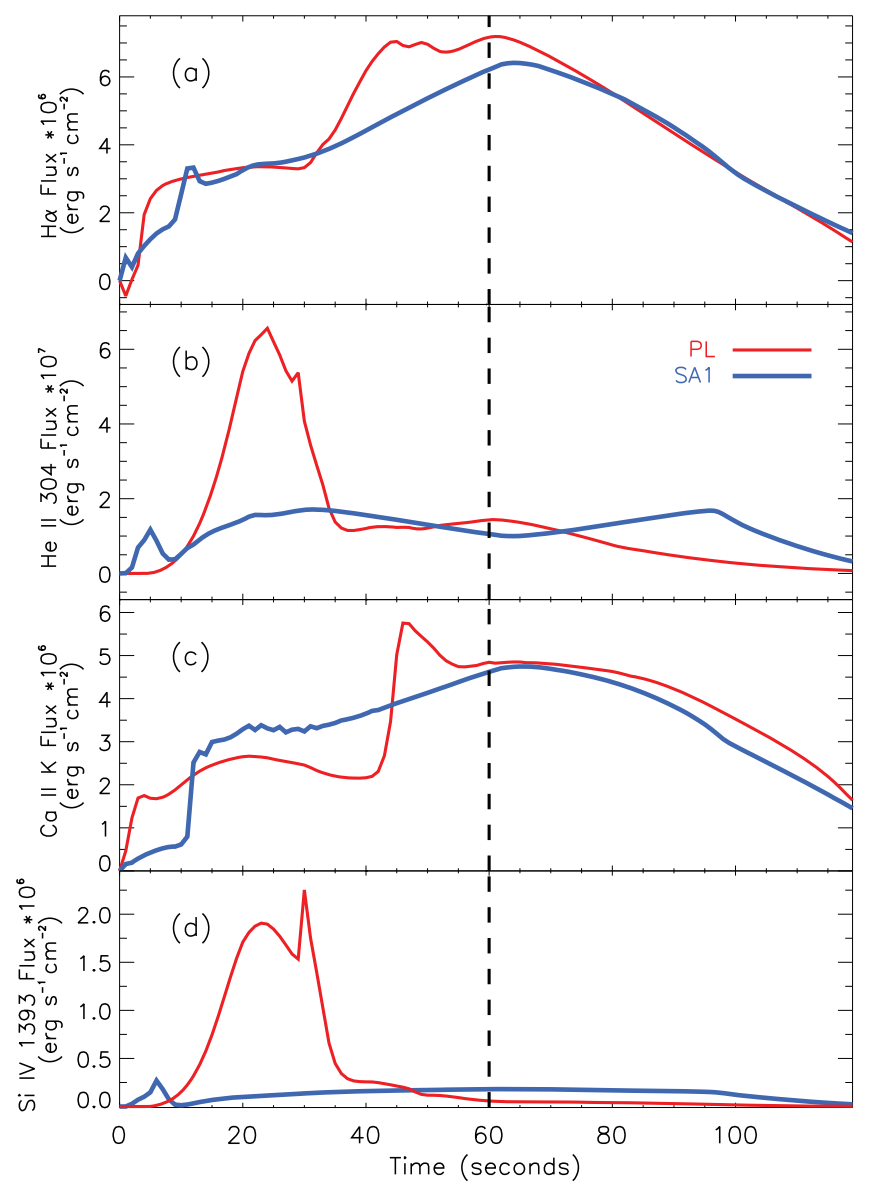

Figure 8. H $\alpha$, He II $304 \AA$, Ca II K, and Si Iv light curves. The emission at the initial time has been subtracted. The dashed line indicates $t_{\max }$.

$304 \AA$ emission is formed by collisional excitation in the quiet Sun, but by the photoionization-recombination process in active regions and during flares. This is consistent with the high-temperature values in the formation region revealed in our simulation.

We note in passing that, as shown in Figure 8, the integrated intensity for the PL model experiences a rapid increase at $t=11 \mathrm{~s}$ and peaks at $23 \mathrm{~s}$, which coincides with an increase in electron density at transition region temperatures. In contrast, such a peak is absent in the light curve of the SA1 model, which exhibits more mild temporal variations.

\subsection{Ca II $K 3934 \AA$ Line Emission}

The Ca II $\mathrm{K}$ line is formed in the transition $2 s-2 p_{1}$, absorbing photons at $3934 \AA$. Vial (1982) modeled the conditions at which the line profile is formed, assuming CRD, requiring low electron densities $\left(2 \times 10^{10} \mathrm{~cm}^{-3}\right)$ and a relatively low ionization degree of hydrogen. Paletou et al. (1993) improved the results by considering the partial frequency redistribution approximation (PRD) in the model, getting higher intensity values. To model the $\mathrm{Ca}$ II $\mathrm{K}$ line profile, it is important to keep in mind that the wings are formed under LTE conditions, while the core is formed under NLTE conditions (Leenaarts et al. 2006). Another point to take into account in the modeling of this line is the formation height during the quiet Sun, which ranges from the chromosphere above the minimum temperature region up to the transition region.
The Ca II K line profile (Figure 12) shows strong asymmetries due to the large plasma velocities at these heights, consistent with that observed by Cauzzi et al. (1993) during the early phase of a solar flare. SA1 presents stronger redshifted profiles (specially at $t=20 \mathrm{~s}$ ) due to its higher velocities than $\mathrm{PL}$, indicating that the $\mathrm{Ca}$ II $\mathrm{K}$ emission is sensitive to plasma velocities.

The monochromatic optical depth (green line in Figure 13) for $\mathrm{Ca}$ II $\mathrm{K}$ shows that the wings of the line are formed at $z=0.41 \mathrm{Mm}$ in both runs, not changing in time. Instead, the core is formed in the transition region, which is located at a lower height for the SA1 model (e.g., see Figure 2). As shown in Figure 13, the contribution function is weaker in the wings than in the line core, where the velocity changes rapidly with height. In fact, the plasma in the formation region is subject to downflows and upflows, which is manifested as the distortion in the line profile near the core. Nevertheless, the wings still exhibit a symmetric shape. We also noted that the $\mathrm{Ca}$ II $\mathrm{K}$ line wings are formed higher in the chromosphere than the $\mathrm{H} \alpha$ line for both runs.

As Figure 8(c) shows, the Ca II $\mathrm{K}$ intensity in the PL model responds faster to the nonthermal electrons than in SA1, reaching higher intensity values at initial times; afterward it follows a similar behavior to the flux of the electrons, $\mathfrak{F}$. The flux for the SA1 model increases almost gradually, starting at $t=12 \mathrm{~s}$ up to $t=65 \mathrm{~s}$, and decreases monotonically afterward.

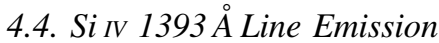

The Si IV $1393 \AA$ line is an optically thin line formed in a narrow region located in the upper chromosphere, which under ionization equilibrium conditions corresponds to a temperature range around $(8 \pm 2) \times 10^{4} \mathrm{~K}$. De Pontieu et al. (2015) showed that the correlation between the nonthermal line broadening and the intensity is reproduced with simulations only when the nonequilibrium ionization is taken into account. This is because the nonequilibrium ionization leads to the presence of $\mathrm{Si}^{3+}$ ions over a much wider range of temperatures than under ionization equilibrium (see, e.g., Olluri et al. 2013).

We synthesized the intensity profile by using the CHIANTI abundances (Dere et al. 1997; Landi et al. 2013) and the plasma properties resulting from our atmosphere. The bottom panels of Figure 14 show the evolution of the Si Iv line profile. This evolution can be described as an initial rapid blueshifted excursion due to chromospheric evaporation of material to the corona, followed by chromospheric compression causing redshifts and then blueshifts again in the late phase.

Comparing the two runs, we note that, in general, PL presents larger blueshifts, while SA1 has greater redshifts, especially early in the simulation. This is due to their different plasma velocity distributions around the $\mathrm{Si}$ IV line formation temperature shown in Figures 4(a) and (b). This trend is qualitatively consistent with the recent simulation result of Testa et al. (2014), which shows that nonthermal electrons are required to produce blueshifted $\mathrm{Si}$ IV emission, while conductive heating alone tends to produce only redshifted emission. The latter case resembles the strong heating in the corona rather than in the lower atmosphere in our Run SA1, owing to the presence of its prominent quasi-thermal component of electrons. We also note differences in the large temporal variations of the intensity between the two runs by up to orders of magnitude, the largest among all four emission lines studied here, as shown in Figures 14 and 8. Early in the 


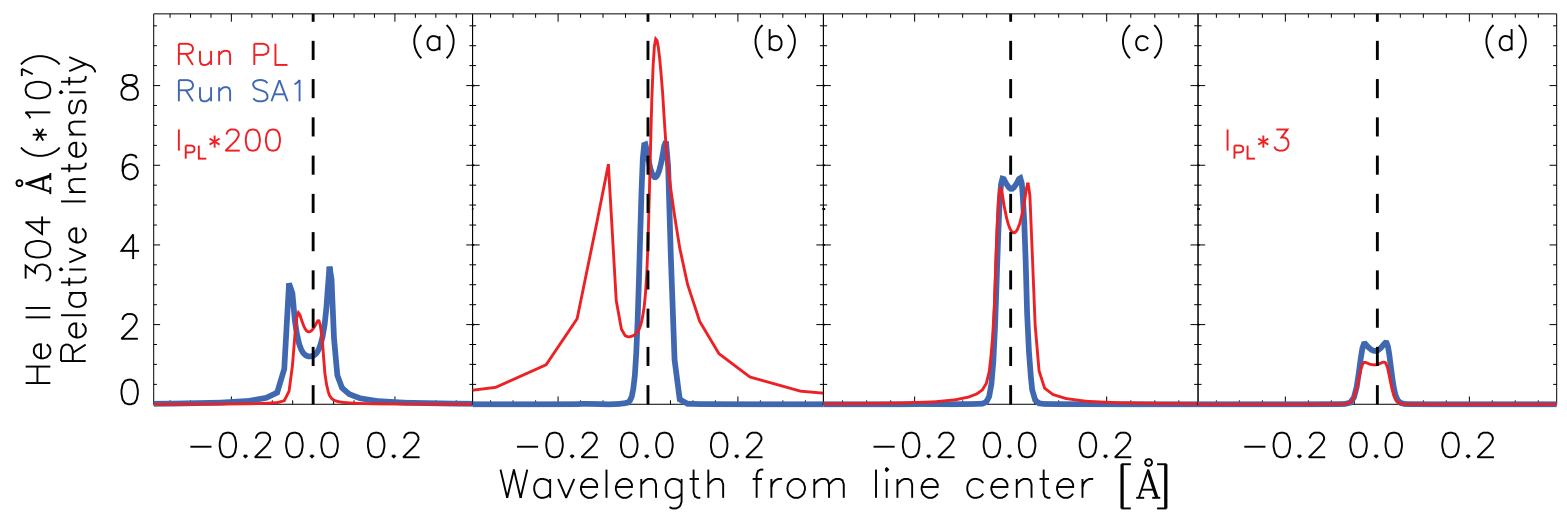

Figure 9. He II $304 \AA$ line profiles at $t=5,20,60$, and $120 \mathrm{~s}$ for runs PL (red line) and SA1 (blue thick line), similarly to Figure 6.
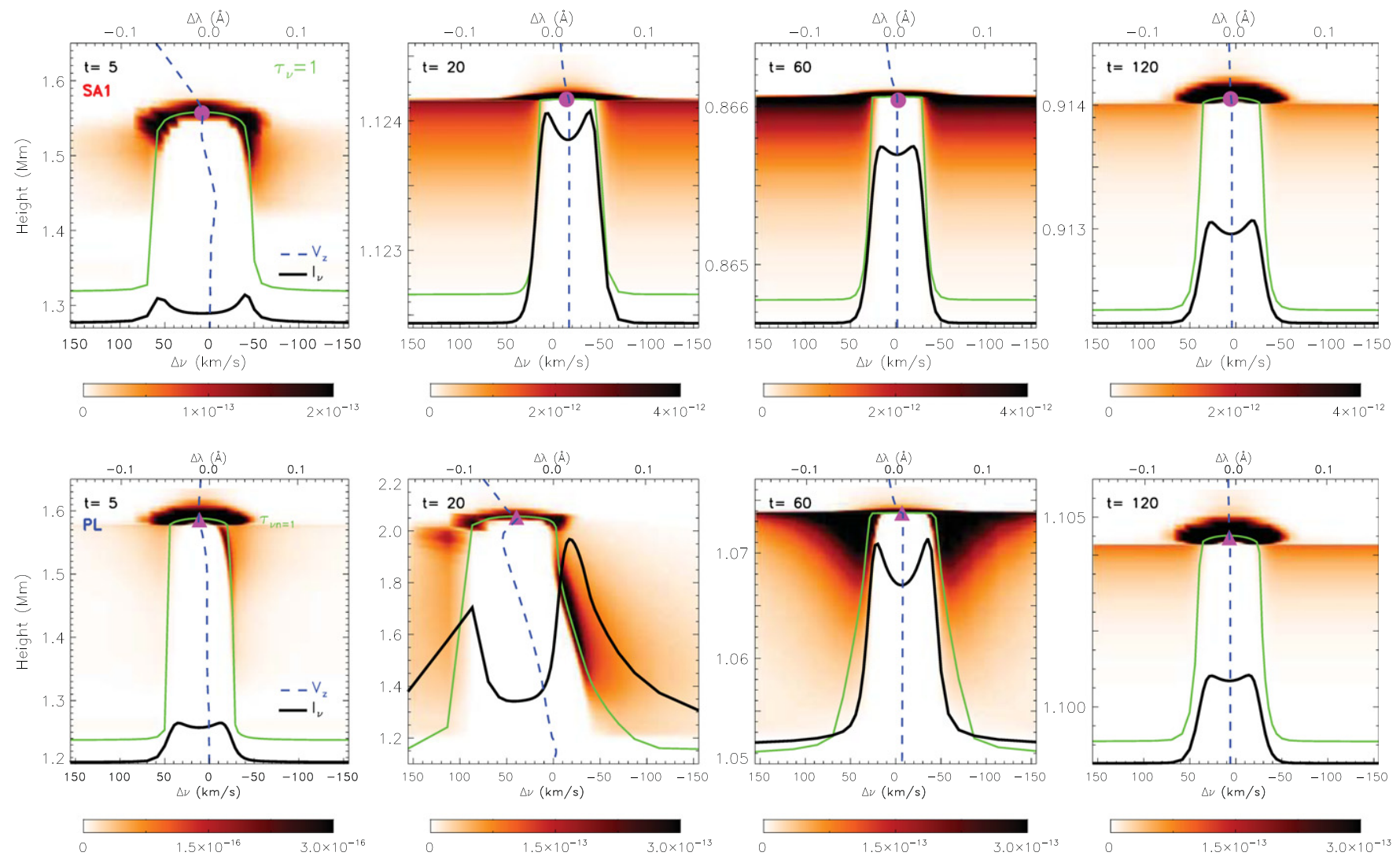

Figure 10. Intensity contribution function for $\mathrm{He}$ II $304 \AA$ as a function of frequency and height, similarly to Figure 7 . Left panel: SA1 Run; right panel: PL Run. The pink circle (in the SA1 case - top) and triangle (in the PL case-bottom) mark the average formation height of the line center. The color scale of each panel is shown in its respective color bar. Note that the line profiles are not in scale.

simulation during $t=20$ and $30 \mathrm{~s}$, PL presents a broad peak in the integrated intensity, more than 7 times higher than that of SA1 (see Figure 8(d)). This is due to its larger electron density hump in the transition region, as shown in Figure 2, and thus greater emission measure.

\section{SUMMARY AND DISCUSSIONS}

The aim of this paper is to investigate the response of the solar atmosphere to the energy input by accelerated electrons. To achieve this, we have extended our earlier study (Paper I) by including radiative transfer calculations. Specifically, we combined the Stanford Unified Acceleration-Transport code with the radiative HD RADYN code. Our primary focus is to compare the results from the more realistic SA model with that from an ad hoc power-law injection model and to obtain synthetic line emissions from our simulation results, which can provide new constraints for the particle acceleration mechanism. Our main findings are the following:

1. In general, the temporal evolution of the atmosphere is determined not only by the energy flux but also by the spectral shape of nonthermal electrons. The results of our Runs PL (power-law injection) and SA1 (SA) are in qualitative agreement with those of Runs $\mathrm{O}$ and $\mathrm{N}$ of Paper I (Liu et al. 2009), respectively. Stochastically accelerated electrons lead to stronger chromospheric evaporation with higher coronal temperatures and plasma velocities (see Table 1) because of their prominent quasi-thermal spectral component. HD shocks form in 

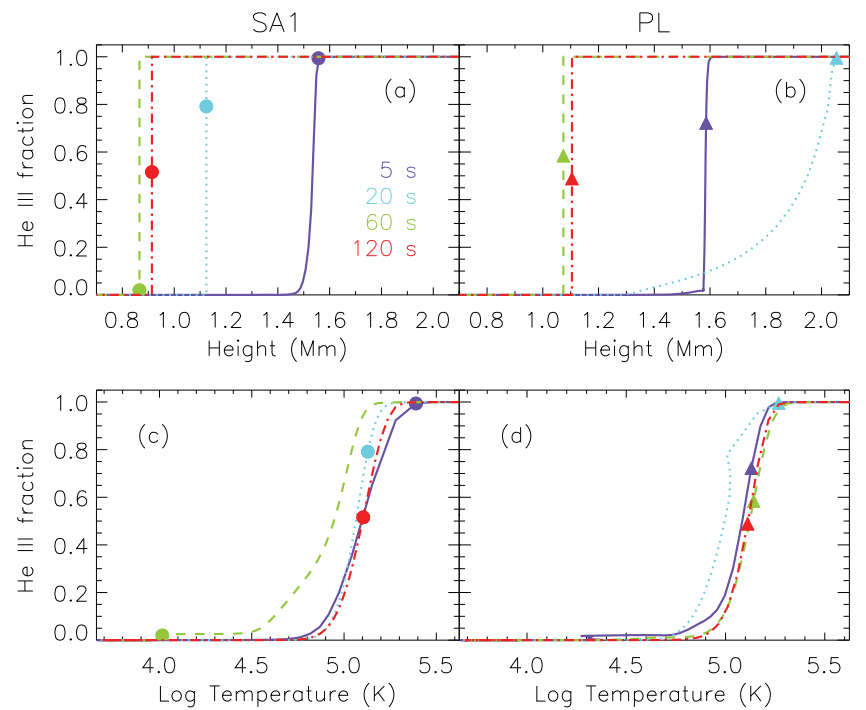

Figure 11. He III ionization fraction as a function of height (top) and temperature (bottom) for Runs SA1 (left) and PL (right) at $t=5,20,60$, and 120 s. The pink circles (in the SA1 case-left) and triangle (in the PL caseright) mark the height and temperature formation of the line center as shown in Figure 10

both cases, lasting longer in PL, but appearing earlier in SA1.

2. The spatial distribution of the electron energy deposition rate per unit volume is concentrated in the upper chromosphere for both runs (see bottom row of Figure 2). In terms of the electron heating rate per unit atmospheric mass, most of the directly deposited energy is radiated away in the chromosphere for Run PL (Figure 15). However, owing to the quasi-thermal spectral component, Run SA1 has a significant amount of energy per unit mass directly deposited in the corona, rather than in the chromosphere (Figure 16). Downward thermal conduction then carries the bulk of this energy to heat the transition region, where radiative loss is not as efficient as in the chromosphere. This is why chromospheric evaporation is much stronger in SA1 than in PL, despite their identical total electron energy flux.

3. In both cases, most of the energy exchange occurs in the lower atmosphere, and most of the net energy gain of the plasma is in the form of thermal energy, which dominates over ionization energy expenses. The thermal energy increase is about two orders of magnitude higher for SA1 than for PL.

4. For different SA runs of different peak electron energy flux $\mathfrak{F}_{\max }$, the maximum upflow velocity increases almost linearly with the $\mathfrak{F}_{\max }$, while the maximum downflow velocity increases at a lower rate (see Appendix B). The temperature peaks at $t_{\max }$ are independent of $\mathfrak{F}_{\max }$.

5. Surprisingly, explosive chromospheric evaporation with upflow speeds of hundreds of kilometers per second is present in our three SA runs with wide-ranging electron energy fluxes $\mathfrak{F}_{\max }$ from $10^{8}$ to $10^{10} \mathrm{erg} \mathrm{s}^{-1} \mathrm{~cm}^{-2}$. In contrast, explosive evaporation only occurred when $\mathfrak{F}_{\text {max }}>3 \times 10^{10} \mathrm{erg} \mathrm{s}^{-1} \mathrm{~cm}^{-2}$ in early simulations by Fisher et al. (1985), who used a power-law injection with a spectral index of 4 and low-energy cutoff of $20 \mathrm{keV}$. Such energy flux was widely quoted as the sole quantity that determines gentle versus explosive evaporation. Our result demonstrates that the shape of the electron spectrum is at least equally, if not more, important as the total energy flux, for the reasons noted above. This is consistent with the conclusions independently reached by Reep et al. (2015) and Allred et al. (2015).

In order to study how the different models affect the energy deposition in the lower chromosphere, we obtained the emission in several wavelength ranges formed at different heights from the upper photosphere to the transition region. According to the line emission for both runs, we find that the emission in $\mathrm{H} \alpha$ and $\mathrm{Ca}$ II $\mathrm{K}$ (two lines that cover the whole chromosphere) seems to be more dependent on the electron flux variation than emission in other wavelengths, following a triangular profile similar to the flux of electrons, $\mathfrak{F}$. The characteristics of the specific emission lines are as follows:

1. The $\mathrm{H} \alpha$ line is a broad line covering the chromosphere, from $0.12 \mathrm{Mm}$ up to the transition region, where the temperature changes drastically. The SA1 Run has higher chromospheric velocities and therefore stronger line profile asymmetries than the PL Run. The core formation is located at a height where the plasma velocity changes drastically (from almost zero in the lower chromosphere to more than $50 \mathrm{~km} \mathrm{~s}^{-1}$ ), covering a broader region for the PL Run. Close to $t_{\max }$ the profile presents a stronger blueshifted peak in the line center and redshifts in the wings, indicating plasma upflows (evaporation) from the chromosphere to the transition region and downflows in the chromosphere. The line intensity is temporally correlated with the electron energy flux, peaking almost at its apex $t_{\text {max }}$.

2. Our synthetic He II $304 \AA$ line is formed within a temperature range from $1 \times 10^{4}$ to $2.5 \times 10^{5} \mathrm{~K}$, which is broader than previously reported for the quiet Sun, when this line is formed by collisional excitation. However, in agreement with the results from Jordan et al. (1993), we find that, for active regions and during flares, the photoionization-recombination processes are more important. Thus, the high temperatures at the formation height in our simulation are not unexpected. This line is formed at the bottom of the transition region, covering a height range between 5 and $300 \mathrm{~km}$ for the PL model, being even narrower for the SA1 model. The shape of the line profile is strongly affected by the plasma velocity at the formation height, showing asymmetries at locations where large velocity gradients are present. The integrated intensity is strongly correlated with the electron density, as manifested in its large enhancement from 11 to $36 \mathrm{~s}$ for the PL model (see Figure 8(b)).

3. The $\mathrm{Ca}$ II $\mathrm{K}$ line is formed in the chromosphere above the temperature minimum, at $0.41 \mathrm{Mm}$ up to the transition region, where the temperature changes drastically. The line profile shows strong asymmetries, consistent with the observations of Cauzzi et al. (1993) during the early phase of a solar flare. The $\mathrm{Ca}$ II $\mathrm{K}$ flux variation responds faster to the nonthermal electron heating in PL than in SA1.

4. The Si IV line is optically thin, formed in the upper chromosphere. Run PL presents stronger chromospheric evaporation, greater up- and downflow velocities, and higher electron density values, resulting in seven times larger Si IV intensity than run SA1. 


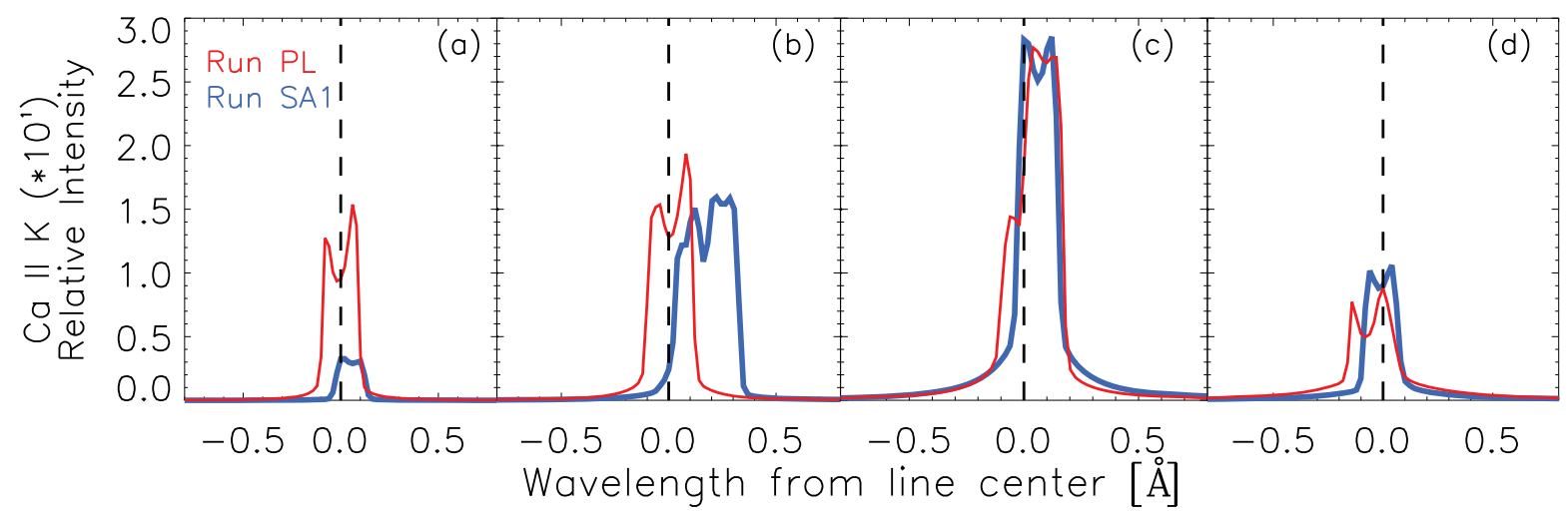

Figure 12. Ca II K $3934 \AA$ Å line profiles at $t=5,20,60$, and $120 \mathrm{~s}$ for runs PL (red line) and SA1 (blue thick line), similarly to Figure 6.
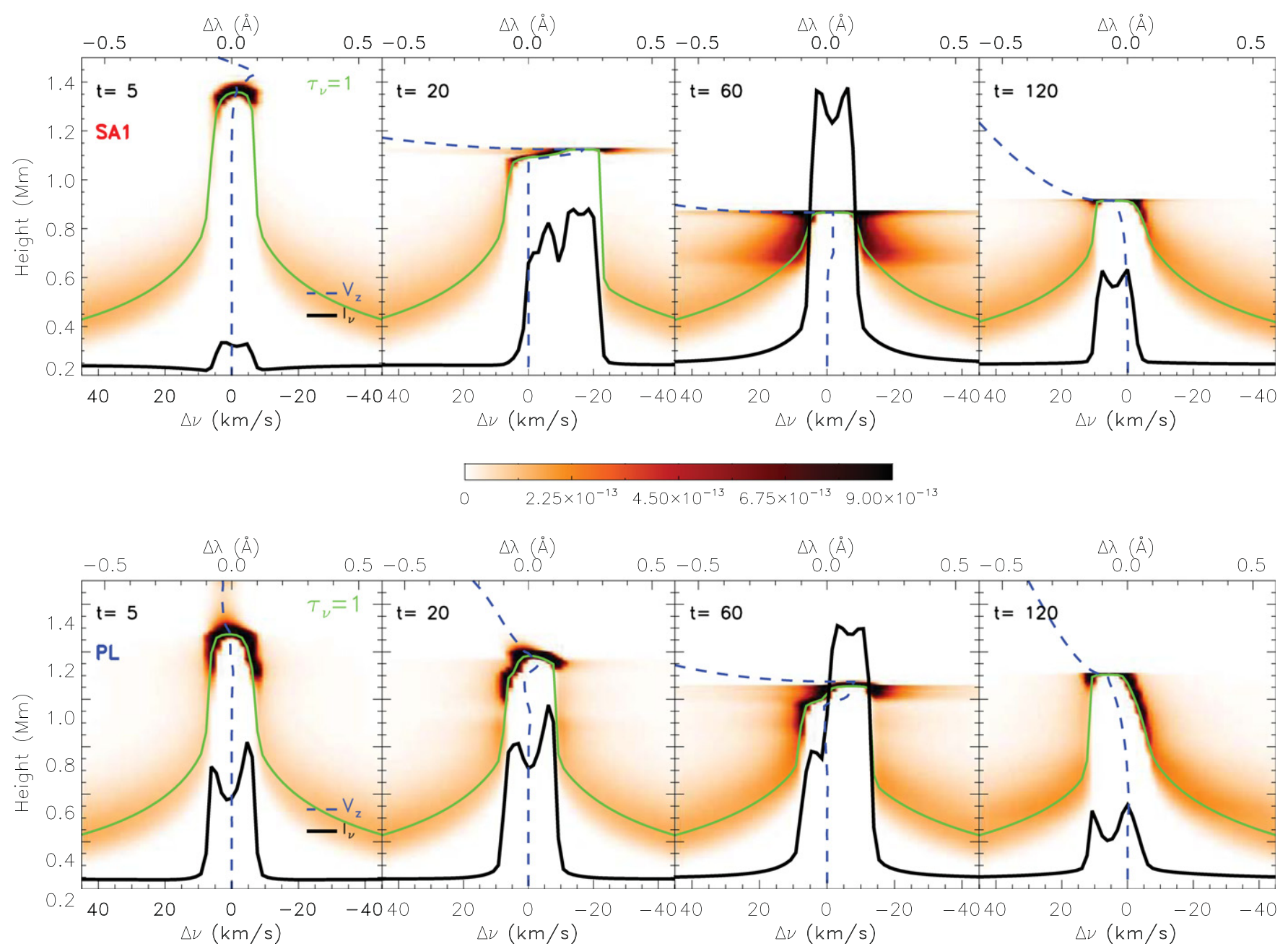

Figure 13. Intensity contribution function for $\mathrm{Ca}$ II $\mathrm{K}$ as a function of frequency and height, similarly to Figure 7. Top panels: SA1 Run; bottom panels: PL Run. All panels have the same color scale as indicated on the color bar.

Our results have demonstrated that the emission-line profiles and light curves of the integrated intensities can provide useful plasma diagnostics and constraints for particle acceleration models. For example, the PL run exhibits similar behaviors of large increases in the light curves of both transition region lines, He II $304 \AA$ and Si IV $1393 \AA$, early during the simulation (see Figure 8). Such large increases are in contrast to the rather slow rise and even drop at certain times in the SA1 case. Such predicted distinction in the temporal evolution can be checked against observations to shed light on the shape of nonthermal electrons.

Our results also have important implications for sunquakes during solar flares (Kosovichev \& Zharkova 1998). Such seismic signals are believed to be caused by the response of the lower atmosphere to impulsive heating by high-energy particles (Kosovichev 2007; Zharkova 2008), among other proposed mechanisms (Lindsey \& Donea 2008; Donea 2011; Fisher et al. 2012). Specifically, sunquakes can be produced by HD 


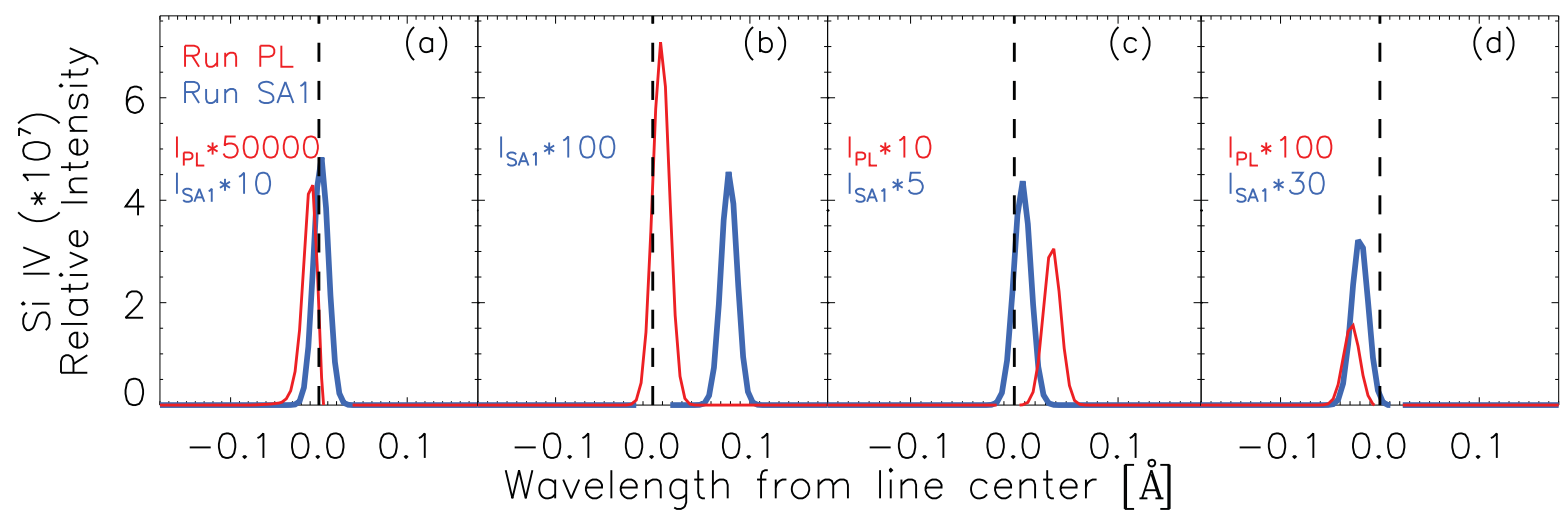

Figure 14. Si Iv $1393 \AA$ Aine profiles at $t=5,20,60$, and $120 \mathrm{~s}$ for runs PL (red line) and SA1 (blue thick line), similarly to Figure 6.
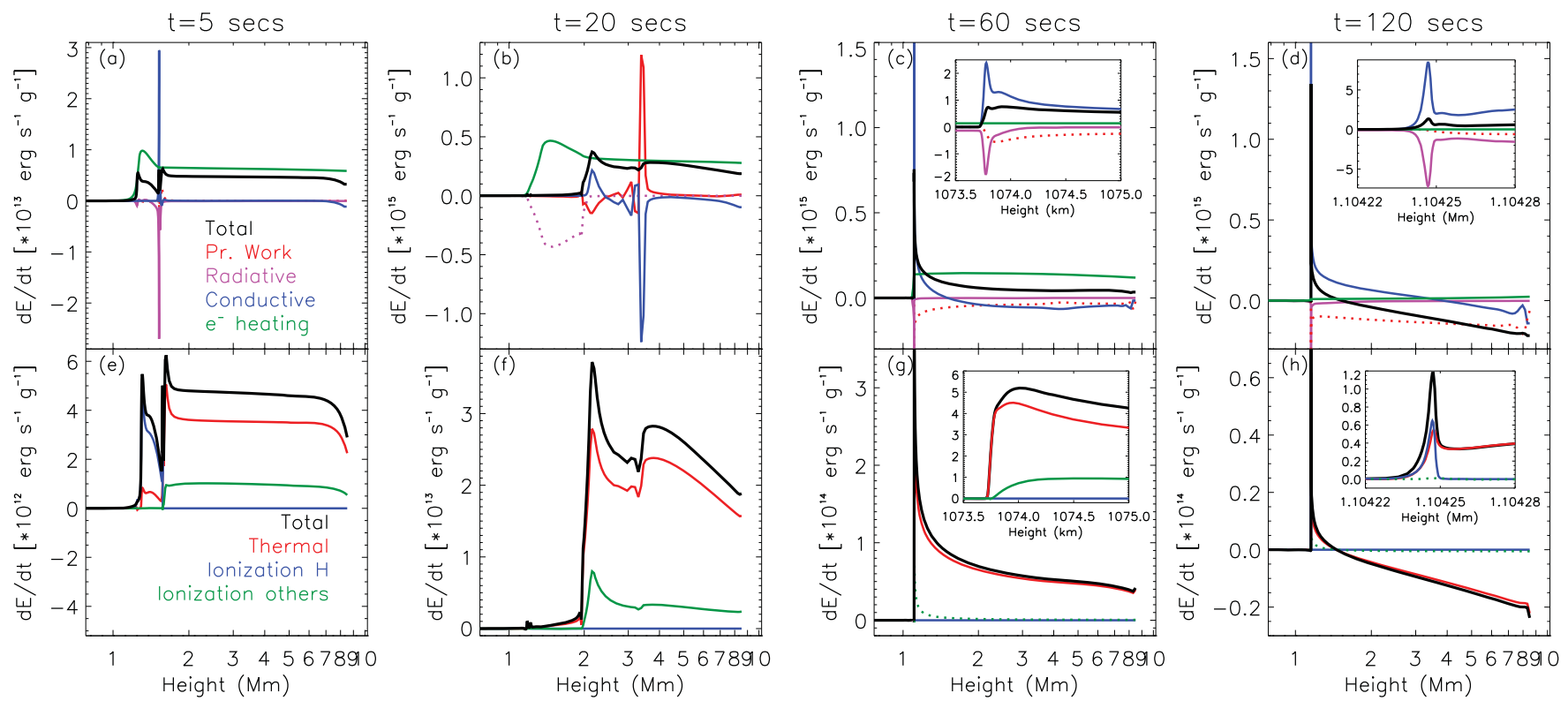

Figure 15. Variations with height of different energy terms for PL at $t=5,20,60$, and $120 \mathrm{~s}$. Note that the $X$-axis is in logarithmic scale. Top: rate of change of major energy terms, including the total internal energy (black thick line), radiative heating (pink), conductive heating (cyan), electron heating (green), and compression work (red). Bottom: rate of change of the total internal energy (black), divided into thermal energy (red), hydrogen ionization and excitation energy (blue), and ionization and excitation energy for elements other than hydrogen (green). The insets show enlarged views near the transition region.

shocks propagating downward from the chromosphere to the photosphere at onsets of flares. In our simulations, we find that the HD response to collisional heating results in initial downflows, especially in the SA1 model, at velocities up to the order of $45 \mathrm{~km} \mathrm{~s}^{-1}$ within the first $10 \mathrm{~s}$ of the flare (see Figure 3(b)). Such high-speed downflows are sufficient to create HD shocks in the lower chromosphere and can be responsible for the sunquakes in the photosphere, as demonstrated in the simulations of Zharkova (2008).

The approach of combined particle acceleration and transport and radiative HD simulation presented in this paper has opened a new chapter in modeling solar flare dynamics. One important future improvement, among others, is to use nonparametric inversion of acceleration parameters from HXR observations (Petrosian \& Chen 2010; Chen \& Petrosian 2013) as inputs to our model. This will allow us to more accurately and realistically simulate the atmospheric evolution, as well as the chromospheric and transition region emission lines, which can be checked against high-resolution spectroscopic observations, such as those from IRIS and DST/ IBIS (e.g., Rubio da Costa et al. 2015). Such comparative investigations will provide promising new constraints to the coupled processes in solar flares, including radiative transfer, $\mathrm{HD}$, and eventually particle acceleration.

This work was supported by NASA LWS grant NNX13AF79G and H-SR grant NNX14AG03G to Stanford University. The research leading to these results has received funding from the European Community's Seventh Framework Program (FP7/2007-2013) under grant agreement no. 606862 (F-CHROMA) and ERC grant agreement no. 291058 (CHROMPHYS). CHIANTI is a collaborative project involving George Mason University, the University of Michigan (USA), and the University of Cambridge (UK). We thank Joel Allred, Tiago Pereira, John Mariska, and Paul Bryans for helpful discussions. W.L. was also supported in part by NASA contract NNG09FA40C (IRIS) to LMSAL and IRIS Guest Investigator grant NNX15AR15G. Resources supporting this work were provided by the NASA High-End Computing (HEC) Program through the NASA Advanced Supercomputing (NAS) Division at Ames Research Center. 

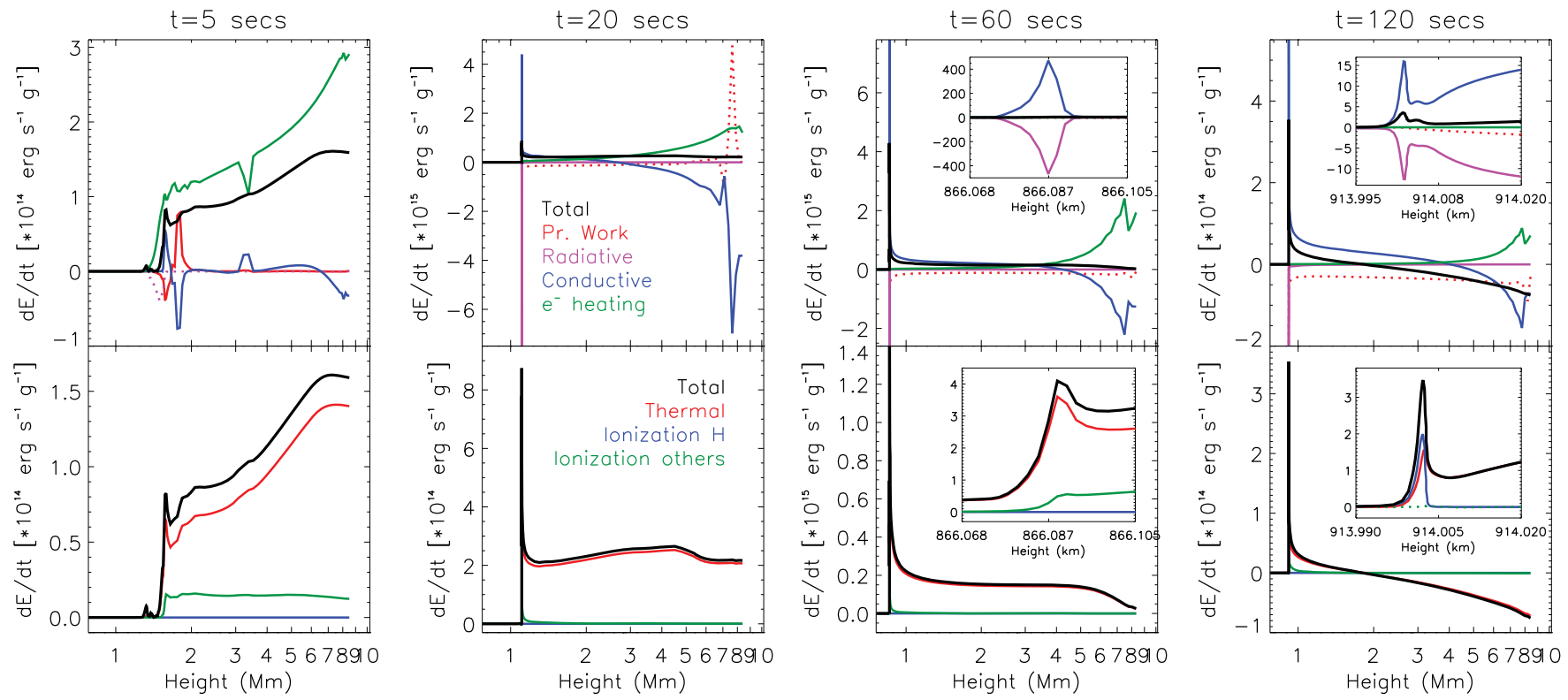

Figure 16. Same as Figure 15, but for Run SA1. Note that the $X$-axis is in logarithmic scale and that at $t=60$ and $120 \mathrm{~s}$, the peaks at the transition region height are truncated in the main panels but are displayed in full in the insets.

\section{APPENDIX A \\ DISTRIBUTION OF ENERGY TERMS}

In this appendix we examine the spatial distribution and temporal evolution of different terms of the energy Equation (2), in order to better understand their contribution to the internal energy of the plasma:

$$
\begin{aligned}
& \frac{\partial \rho \epsilon}{\partial t}+\frac{\partial \rho v \epsilon}{\partial z}+\left(p+q_{v}\right) \frac{\partial v}{\partial z} \\
& +\frac{\partial}{\partial z}\left(F_{c}+F_{\mathrm{r}, \text { thin }}+F_{\mathrm{r}, \text { detail }}\right)-Q_{\text {init }}-Q_{e}-Q_{\mathrm{XEUV}}=0 .
\end{aligned}
$$

Here $z$ is distance, $t$ time, $\rho$ density, $v$ velocity, $p$ pressure, $\epsilon$ internal energy per unit mass, $q_{v}$ the viscous stress, and $F_{c}$ and $F_{r}$ conductive and radiative fluxes. The specific energy terms include viscous heating $-q_{v}(\partial v / \partial z)$, compression work $-p(\partial v / \partial z)$, conductive heating $-\partial F_{c} / \partial z$, the heating function $Q_{\text {init }}$ to create the initial atmosphere, collisional heating $Q_{e}$ by nonthermal electrons, X-ray plus EUV heating $Q_{\mathrm{XEUV}}$, the thin radiative heating $-\partial F_{\mathrm{r}, \text { thin }} / \partial z$ of elements treated in LTE, and the radiative flux $-\partial F_{\mathrm{r}, \text { detail }} / \partial z$ calculated from solving the radiative transfer equation. Here $F_{\mathrm{r} \text {,detail }}$ is integrated over wavelength and includes about 34,000 wavelengths in the temperature range between 0.1 and $10 \mathrm{MK}$, covering the continua from 1 to $40000 \AA$ and spectral lines of hydrogen, helium, calcium, and magnesium, treated in NLTE.

Since the atmospheres evolve differently in the two runs, we discuss them individually. In order to avoid complex graphics, here we show only the energy terms with significant contributions to the total internal energy.

\section{A.1. PL Run}

The top panels of Figure 15 show the rate of change of different energies per unit mass (cf. heating rate per unit volume in the fourth row of Figure 2): internal energy (black thick line), radiative heating (pink), conductive heating (cyan), electron heating (green), and compression work (red). Most of the energy exchange occurs in the lower atmosphere, and the main contribution to the internal energy change is the electron heating rate. At $t=5 \mathrm{~s}$ and $z=1.55 \mathrm{Mm}$ (within the chromosphere), the conductive and electron heating rates are the main source of heating, which are mostly balanced by the radiative loss term, explaining the small temperature changes at this time (see Figure 2).

At $t=20 \mathrm{~s}$, deep in the chromosphere, electron heating $Q_{e}$ is largely balanced by radiative losses. However, in the transition region (near $z=2 \mathrm{Mm}$ ) and corona, it dominates over the loss terms and results in an increase in the internal energy and temperature. Around the chromospheric evaporation front at $z=3.7 \mathrm{Mm}$, the pressure work due to the negative velocity gradient leads to a local temperature enhancement (see Figure 2), thus compensating conductive cooling.

At later times, conductive heating in the transition region becomes important. For example, at $t=60 \mathrm{~s}$ and $z=1.07 \mathrm{Mm}$, the conductive heating rate increases in a height range of less than $150 \mathrm{~m}$ from almost zero to $3 \times 10^{15}\left(\mathrm{erg} \mathrm{s}^{-1} \mathrm{~g}^{-1}\right)$. This is also the region where most of the nonthermal electron energy is deposited (see bottom row of Figure 2).

The bottom panel of Figure 15 shows the change rate of the total internal energy (black thick line) divided into the thermal energy of the material (red), the sum of ionization energy of hydrogen (blue), and the rest of the constituent atoms (green). As can be seen, the thermal energy change dominates at all times, but the total ionization energy of atoms other than hydrogen is important at early times.

\section{A.2. SAl Run}

In the SA1 model, the electron heating rate per unit mass, the major contributor to the total internal energy increase (see Figure 16), is primarily located in the corona, rather than the transition region or chromosphere as in the case of the PL model. This results in the significant increase of the coronal temperature (see Figure 2). At $t=5 \mathrm{~s}$ the pressure work balances the conductive cooling at $z=1.86 \mathrm{Mm}$ (above the transition region), where the electron density is higher. 


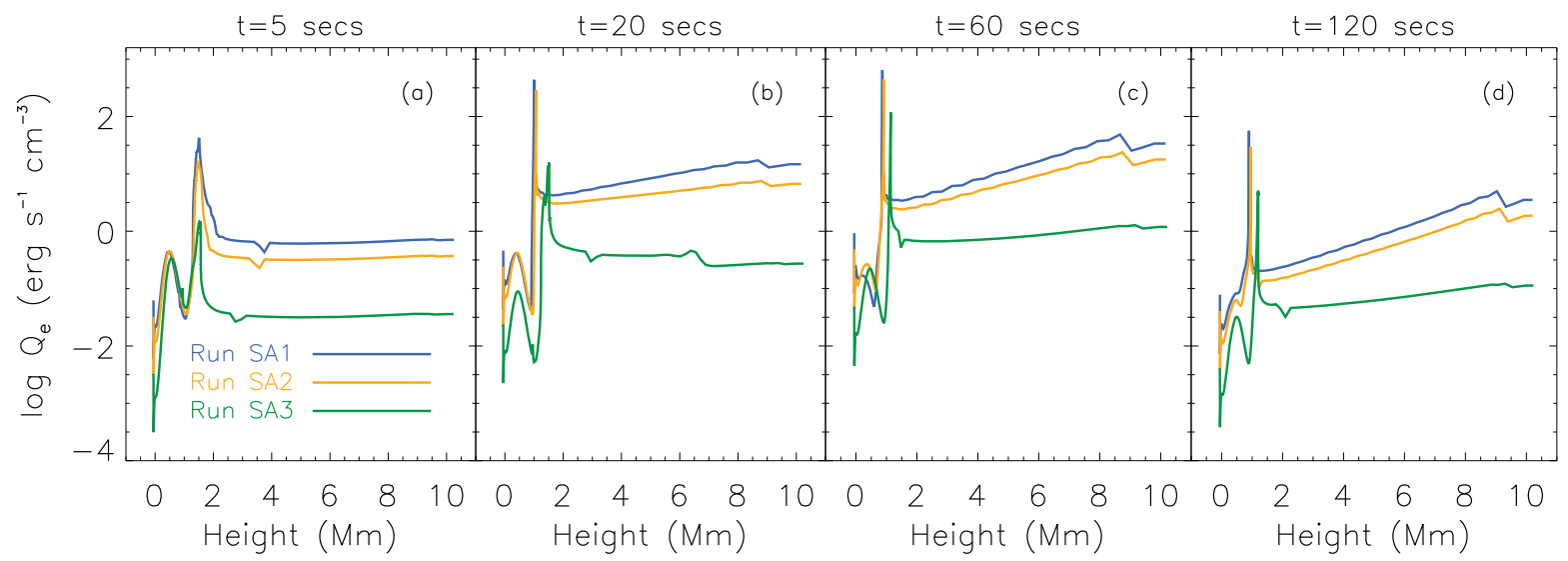

Figure 17. Spatial distribution of the electron heating rate $Q_{e}(z)$ for Runs SA1, SA2, and SA3 (see Table 1) at selected times, $t=5,20,60$, and $120 \mathrm{~s}$.

At $t=20 \mathrm{~s}$ the bulk of pressure work has moved to the upper corona and is still balanced by conductive cooling. At $z=1.125 \mathrm{Mm}$, where the transition region is located, the conductive heating rate increases sharply up to $4.4 \times 10^{15}$ $\left(\mathrm{erg} \mathrm{s}^{-1} \mathrm{~g}^{-1}\right.$ ) and is partly radiated away and balanced by the pressure work within a very narrow region, where the nonthermal electron energy is mostly deposited (see bottom row of Figure 2). The electron heating in the lower atmosphere is negligible in comparison with the other terms, and it is mostly radiated away.

As the atmosphere evolves, most of the conductive energy in the transition region is radiated away, and very little is left to heat the plasma. This energy exchange occurs in a very narrow layer, less than $10 \mathrm{~m}$ thick, at $\approx 0.866 \mathrm{Mm}$, where the conductive heating rate increases from almost 0 to $5 \times 10^{17} \mathrm{erg} \mathrm{s}^{-1} \mathrm{~g}^{-1}$ (see the inset in Figure 16(c)). The atmosphere is already ionized in this region and does not radiate efficiently anymore, thus increasing the total internal energy and temperature. Note that for a better comparison, the last two columns of Figure 16 do not show the total length of the peak.

As shown in the bottom panels, most of the total energy gain (black thick line) is used to increase the thermal energy (red line), as in the case of PL. However, these energy change rates are one to two orders of magnitude higher than those in PL.

\section{APPENDIX B EFFECTS OF THE AMPLITUDE OF THE ELECTRON FLUX $\mathfrak{F}_{\max }$}

Here we present the result of SA runs of different peak energy fluxes of the nonthermal electrons, $\mathfrak{F}_{\max }$, but with the same triangular-shaped temporal profile of $\mathfrak{F}(t)$ as in Runs SA1 and PL. The characteristics of these runs are shown in Table 1. As expected, a higher electron flux leads to higher maximum upflow and downflow speeds and temperatures that occur at earlier times. The only exception is the maximum temperature that almost always occurs at the time of $\mathfrak{F}_{\max }$, i.e., $t=60 \mathrm{~s}$. A factor of 20 increase in $\mathfrak{F}_{\max }$ from $5.7 \times 10^{8} \mathrm{erg} \mathrm{s}^{-1} \mathrm{~cm}^{-2}$ (Run SA3) to $1.2 \times 10^{10} \mathrm{erg} \mathrm{s}^{-1} \mathrm{~cm}^{-2}$ (Run SA1) only results in a factor of two increase in the maximum upflow speed $v_{\max }$ from 377 to $750 \mathrm{~km} \mathrm{~s}^{-1}$, a factor of $50 \%$ increase in the maximum downflow speed $v_{\min }$ from -28 to $-41 \mathrm{~km} \mathrm{~s}^{-1}$, and a factor of three increase in the maximum temperature from 8 to $23 \mathrm{MK}$. These results are in line with those of Liu (2008); see his Table 7.1 for simulations using the same spectrum of stochastically accelerated electrons as we have adopted here.

Fisher et al. (1985) found that an electron energy flux of $<10^{10} \mathrm{erg} \mathrm{s}^{-1} \mathrm{~cm}^{-2}$ can produce so-called gentle chromospheric evaporation with upflow speeds of tens of kilometers per second, while an energy flux of $>3 \times 10^{10}$ $\mathrm{erg} \mathrm{s}^{-1} \mathrm{~cm}^{-2}$ would produce "explosive" evaporation with upflow speeds of hundreds of kilometers per second and downflow speeds of tens of kilometers per second. As shown in Table 1, all the SA runs have energy flux below this threshold, and yet all exhibit characteristics of "explosive" evaporation. This demonstrates that the energy flux is not the only factor that dictates the atmospheric response to electron heating; the electron spectral shape is also critical because it determines the spatial distribution of the energy deposition. The key difference is that Fisher et al. (1985) injected a single power-law electron spectrum of an index of $\delta=4$ and cutoff energy of $20 \mathrm{keV}$, which is comparable to that in our PL run. Such nonthermal electrons directly deposit most of their energy deep in the chromosphere, which is largely radiated away (see Figure 15). In our SA runs, the electron spectrum has a prominent quasi-thermal component and thus results in heating primarily in the corona (in terms of heating rate per unit mass; see Figure 16). Then thermal conduction becomes the primary heating agent in the lower atmosphere, mainly in the transition region where radiative loss is not as strong as in the chromosphere. As a result, there is relatively more energy left to heat the plasma and drive chromospheric evaporation. This is similar to the case in Paper I, although radiative transfer was not included there. This result indicates that the stochastically accelerated electrons, because of their preferential heating in the corona rather than directly in the chromosphere, are more efficient in driving chromospheric evaporation than power-law electrons with a cutoff energy. This also indicates that, in addition to direct collisional heating by nonthermal electrons as commonly believed, thermal conduction can play an important role in driving chromospheric evaporation, as observed in some flares (e.g., Zarro \& Lemen 1988; Battaglia et al. 2009). This is even more so for weak flares, such our SA3 run.

In the case of faint flares (Run SA3), most of the electron heating rate, $Q_{e}$, is transformed into conductive heating and radiated away along the atmosphere, explaining why the electron heating in Figure 17 (green line) remains almost constant in the corona. By increasing the electron flux $\mathfrak{F}$, in 


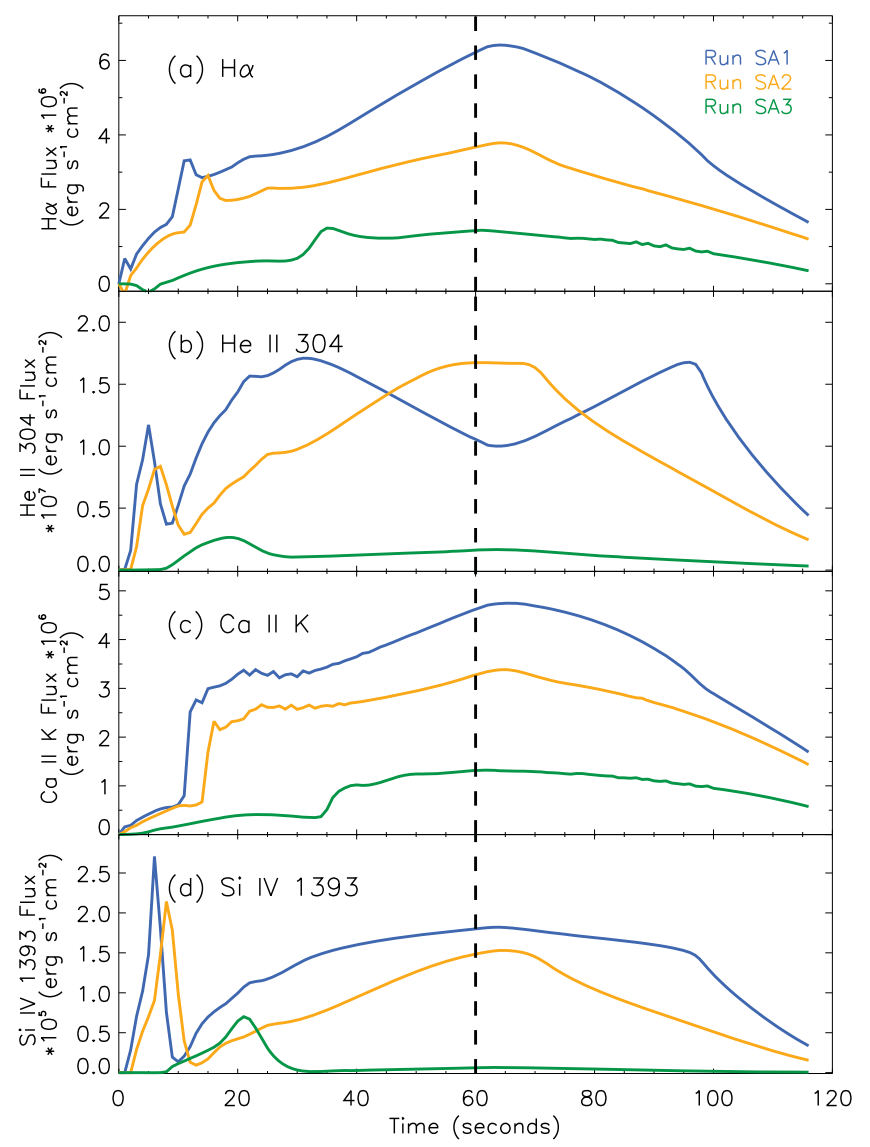

Figure 18. Light curves of the $\mathrm{H} \alpha 6563 \AA$, He II $304 \AA$, Ca II K $3934 \AA$, and Si Iv $1393 \AA$ Aines for Runs SA1-SA3. The emission at the initial time has been subtracted. The vertical dashed line indicates $t_{\max }$.

Runs SA1 and SA2 (blue and yellow lines), the heating increases at the top of the loop with time.

\section{B.1. Emission Lines}

Figure 18 shows the light curve of the $\mathrm{H} \alpha$, He II $304 \AA$, Ca II K, and Si IV $1393 \AA$ lines for the three SA models, where the emission of the quiet Sun (at the initial time) has been removed. In general, for higher peak electron energy fluxes, the line intensity increases faster with time, because of more rapid HD response of the atmosphere, and reaches higher peak values. This is the case for the $\mathrm{H} \alpha$ and $\mathrm{Ca}$ II $\mathrm{K}$ fluxes, which are positively correlated with the electron flux, peaking around $t_{\text {max }}$. However, the He II $304 \AA$ intensity in Run SA1 decreases in the middle portion of the flare duration, showing anticorrelation in time with the electron flux. Each of the He II light curves of the three runs also shows distinct behaviors. In Table 1 we can see that the maximum temperature decreases linearly with the flux of the injected electrons. Therefore, the recombination-photoionization processes may play a less important role in the formation of the line, giving preference to the collision excitation processes. This may explain why the shape of the He II $304 \AA$ line profiles and the flux changes with $\mathfrak{F}$.

The temporal evolution of the Si IV $1393 \AA$ flux shows an earlier bump associated with the increase of the electron density in the chromosphere and transition region. As discussed in Section 4.4, an increase of the electron density leads to an increase of the emission measure at this wavelength range and thus the increase in intensity of this optically thin line.

\section{REFERENCES}

Abbett, W. P., \& Hawley, S. L. 1999, ApJ, 521, 906

Allred, J. C., Hawley, S. L., Abbett, W. P., \& Carlsson, M. 2005, ApJ, 630, 573

Allred, J. C., Kowalski, A. F., \& Carlsson, M. 2015, ApJ, 809, 104

Andretta, V., Del Zanna, G., \& Jordan, S. D. 2003, A\&A, 400, 737

Battaglia, M., Fletcher, L., \& Benz, A. O. 2009, A\&A, 498, 891

Carlsson, M., \& Stein, R. F. 1992, ApJL, 397, L59

Carlsson, M., \& Stein, R. F. 1997, ApJ, 481, 500

Cauzzi, G., Falchi, A., Falciani, R., \& Smaldone, L. A. 1993, AdSpR, 13, 311 Chen, Q., \& Petrosian, V. 2013, ApJ, 777, 33

De Pontieu, B., McIntosh, S., Martinez-Sykora, J., Peter, H., \& Pereira, T. M. D. 2015, ApJL, 799, L12

De Pontieu, B., Title, A. M., Lemen, J. R., et al. 2014, SoPh, 289, 2733

Dere, K. P., Landi, E., Mason, H. E., Monsignori Fossi, B. C., \& Young, P. R. 1997, A\&As, 125, 149

Donea, A. 2011, SSRv, 158, 451

Dorfi, E. A., \& Drury, L. O. 1987, JCoPh, 69, 175

Emslie, A. G. 1978, ApJ, 224, 241

Emslie, A. G. 1981, ApJ, 249, 817

Fisher, G. H., Bercik, D. J., Welsch, B. T., \& Hudson, H. S. 2012, SoPh, 277, 59

Fisher, G. H., Canfield, R. C., \& McClymont, A. N. 1985, ApJ, 289, 414

Golding, T. P., Carlsson, M., \& Leenaarts, J. 2014, ApJ, 784, 30

Gustafsson, B. 1973, A FORTRAN Program for Calculating "Continuous" Absorption Coefficients of Stellar Atmospheres (Uppsala: Lalidstingets Verkstader)

Hamilton, R. J., \& Petrosian, V. 1992, ApJ, 398, 350

Holman, G. D. 1985, ApJ, 293, 584

Hoyng, P., Duijveman, A., Machado, M. E., et al. 1981, ApJL, 246, L155

Johannesson, A., Marquette, W., \& Zirin, H. 1995, SoPh, 161, 201

Johannesson, A., Marquette, W. H., \& Zirin, H. 1998, SoPh, 177, 265

Jordan, C. 1975, MNRAS, 170, 429

Jordan, S. D., Thompson, W. T., Thomas, R. J., \& Neupert, W. M. 1993, ApJ, 406, 346

Kašparová, J., Varady, M., Heinzel, P., Karlický, M., \& Moravec, Z. 2009, A\&A, 499, 923

Kosovichev, A. G. 2007, ApJL, 670, L65

Kosovichev, A. G., \& Zharkova, V. V. 1998, Natur, 393, 317

Kundu, M. R., White, S. M., Gopalswamy, N., \& Lim, J. 1994, ApJS, 90, 599

Laming, J. M., \& Feldman , U. 1992, ApJ, 386, 364

Landi, E., Young, P. R., Dere, K. P., Del Zanna, G., \& Mason, H. E. 2013, ApJ, 763, 86

Leach, J., \& Petrosian, V. 1981, ApJ, 251, 781

Leenaarts, J., Carlsson, M., \& Rouppe van der Voort, L. 2012, ApJ, 749, 136

Leenaarts, J., Rutten, R. J., Sütterlin, P., Carlsson, M., \& Uitenbroek, H. 2006, A\&A, 449, 1209

Lin, R. P. 1985, SoPh, 100, 537

Lindsey, C., \& Donea, A.-C. 2008, SoPh, 251, 627

Liu, S., Petrosian, V., \& Mason, G. M. 2004, ApJL, 613, L81

Liu, S., Petrosian, V., \& Mason, G. M. 2006, ApJ, 636, 462

Liu, W. 2008, Solar Flares as Natural Particle Accelerators: A High-Energy View from X-ray Observations and Theoretical Models (Saarbrücken, Germany: VDM Verlag)

Liu, W., Chen, Q., \& Petrosian, V. 2013, ApJ, 767, 168

Liu, W., Petrosian, V., \& Mariska, J. T. 2009, ApJ, 702, 1553

Mariska, J. T., Emslie, A. G., \& Li, P. 1989, ApJ, 341, 1067

McTiernan, J. M., \& Petrosian, V. 1990, ApJ, 359, 524

Milkey, R. W., \& Mihalas, D. 1973, ApJ, 185, 709

Miller, J. A. 1997, ApJ, 491, 939

Milligan, R. O., \& Dennis, B. R. 2009, ApJ, 699, 968

O’Dwyer, B., Del Zanna, G., Mason, H. E., Weber, M. A., \& Tripathi, D. 2010 , A\&A, 521, A21

Olluri, K., Gudiksen, B. V., \& Hansteen, V. H. 2013, ApJ, 767, 43

Paletou, F., Vial, J.-C., \& Auer, L. H. 1993, A\&A, 274, 571

Park, B. T., \& Petrosian, V. 1995, ApJ, 446, 699

Petrosian, V. 2012, SSRv, 173, 535

Petrosian, V., \& Chen, Q. 2010, ApJL, 712, L131

Petrosian, V., \& Donaghy, T. Q. 1999, ApJ, 527, 945

Petrosian, V., Donaghy, T. Q., \& McTiernan, J. M. 2002, ApJ, 569, 459

Petrosian, V., \& East, W. E. 2008, ApJ, 682, 175

Petrosian, V., \& Liu, S. 2004, ApJ, 610, 550 
Pötzi, W., Temmer, M., Veronig, A., Hirtenfellner-Polanec, W., \& Baumgartner, D. 2013, EGUGA, 15, 1459

Ramaty, R. 1979, in AIP Conf. Ser. 56, Particle Acceleration Mechanisms in Astrophysics, ed. J. Arons, C. McKee \& C. Max (Melville, NY: AIP), 135

Reep, J., Bradshaw, S., \& Alexander, D. 2015, arXiv:1506.08115

Rubio da Costa, F., Kleint, L., Petrosian, V., Sainz Dalda, A., \& Liu, W. 2015, ApJ, 804, 56

Sakao, T. 1994, PhD thesis, Univ. Tokyo

Sironi, L., \& Spitkovsky, A. 2009, ApJL, 707, L92
Švestka, Z. 1976, in Several Solar Aspects of Flare-Associated Particle, ed. P. S. McIntosh \& M. Dryer (Moskva: Mir.), 141

Testa, P., De Pontieu, B., Allred, J., et al. 2014, Sci, 346, B315

Tsuneta, S., \& Naito, T. 1998, ApJL, 495, L67

Vial, J. C. 1982, ApJ, 253, 330

Woods, T. N., Eparvier, F. G., Hock, R., et al. 2012, SoPh, 275, 115

Zarro, D. M., \& Lemen, J. R. 1988, ApJ, 329, 456

Zharkova, V. V. 2008, SoPh, 251, 641

Zirin, H. 1988, Astrophysics of the Sun (Cambridge: Cambridge Univ. Press) 